\title{
Supervisory Control of Fuzzy Discrete Event Systems: A Formal Approach*
}

\footnotetext{
${ }^{*}$ This work was supported in part by the National Natural Science Foundation under Grant 90303024 and the Guangdong Province Natural Science Foundation under Grant 020146 and Grant 031541 of China.

${ }^{\dagger}$ D. Qiu is with Department of Computer Science, Zhongshan University, Guangzhou, 510275, China (email: issqdw@mail.sysu.edu.cn).
} 
Abstract: Fuzzy discrete event systems (DESs) were proposed recently by Lin and Ying [19], which may better cope with the real-world problems with fuzziness, impreciseness, and subjectivity such as those in biomedicine. As a continuation of [19], in this paper we further develop fuzzy DESs by dealing with supervisory control of fuzzy DESs. More specifically, (i) we reformulate the parallel composition of crisp DESs, and then define the parallel composition of fuzzy DESs that is equivalent to that in [19]; max-product and max-min automata for modeling fuzzy DESs are considered; (ii) we deal with a number of fundamental problems regarding supervisory control of fuzzy DESs, particularly demonstrate controllability theorem and nonblocking controllability theorem of fuzzy DESs, and thus present the conditions for the existence of supervisors in fuzzy DESs; (iii) we analyze the complexity for presenting a uniform criterion to test the fuzzy controllability condition of fuzzy DESs modeled by max-product automata; in particular, we present in detail a general computing method for checking whether or not the fuzzy controllability condition holds, if max-min automata are used to model fuzzy DESs, and by means of this method we can search for all possible fuzzy states reachable from initial fuzzy state in max-min automata; also, we introduce the fuzzy $n$-controllability condition for some practical problems; (iv) a number of examples serving to illustrate the applications of the derived results and methods are described; some basic properties related to supervisory control of fuzzy DESs are investigated. To conclude, some related issues are raised for further consideration.

Index Terms: Discrete event systems, controllability, supervisory control, fuzzy systems, fuzzy finite automata, computing with words.

\section{Introduction}

A Discrete Event System (DES) is a discrete-state, event-driven dynamical system in which the state space is described by a discrete set, and states evolve in terms of asynchronous occurrence of discrete events over time $[11,1,26,27]$. Actually, DESs represent many technological and engineering systems such as communication networks, computer networks, manufacturing systems, and transportation systems. So far, many modeling approaches of DESs have been proposed and developed, including the notably finite automata [26,11,1,31], Petri nets $[22,7,1]$, and their variations [1]. 
In DESs, an important research direction is the supervisory control, which was launched by Ramadge and Wonham $[26,27]$ in the 1980 's. Since then, many researchers have also made contributions to the developments and applications of DES theory (for example, [11,1,31] and the references therein). In the study of supervisory control, a DES can be well modeled by a formal language of finite strings of event symbols, and the set of all possible events is partitioned into both controllable and uncontrollable events. In this framework, a supervisory mechanism is established, in which the supervisor may enable or disable the controllable events in order to satisfy a set of qualitative specifications on the admissible occurrences of the events that can be executed by the system. Moreover, the supervisor may not be able to observe all of the events generated by the system due to the existence of unobservable events in the set of events.

In order to understand more intuitively supervisory control of crisp DESs under complete observation (all events can be seen), let us further introduce it more formally. (For the details, we refer to $[1,11]$.) Here we assume that the given DES is modeled by a finite automaton $G$. The behavior of the DES is characterized by the language $L(G)$ generated by $G$, but the behavior is not satisfactory, that is, $L(G)$ contains strings that are not allowed. Thus, our objective is to consider the sublanguages of $L(G)$ that stand for the legal or admissible behavior for the controlled system. For example, if $K \subseteq L(G)$ is a legal sublanguage of $L(G)$, then we attempt to design a supervisor, denoted by $S$, such that $S$ interacting $G$ will result in the behavior desired. In general, $S$ has the capability of disabling some feasible events of $G$, and the set of feasible events is termed as controllable events. If $S$ exists, then $K$ is called to be controllable; otherwise, we hope to find the largest controllable sublanguage and the smallest controllable superlanguage of $K$. Indeed, computing for finding these sublanguages is related to classical formal language and automata theory $[10,1,11]$.

Though most of the research on supervisory control of DESs has focused on systems that are modeled as deterministic finite automata, there are also interesting studies concerning nondeterministic DESs [8] and probabilistic DESs (see [16] and the references therein) for dealing with some uncertainty problems and complex information and systems in practice. However, in real-life situation, there are also a large number of problems with vagueness, impreciseness, and subjectivity that can be reasonably described by the use of words instead of numbers, where words mean the possibility distributions suggested by Zadeh [38], that is, fuzzy subsets. For example, "young", "old", "small", "large", "excellent", "good", and "poor", etc. can be viewed as the words that are possibility distributions. Actually, comput- 
ing with words, as a methodology, was advocated by Zadeh [38,39] and has been significantly developed by others [40], which may conform more to human's perception when describing the real-world problems. Therefore, recently, Lin and Ying $[18,17]$ initiated significantly the study of fuzzy DESs by combining fuzzy set theory with DESs, with a desire to solve those problems not capable of being satisfactorily treated by conventional DESs.

Exactly, there are a large number of DESs to be treated better in a manner of computing with words when describing their states. This is especially true in biomedical applications, as stressed in [19]. For example, if we describe the health condition of a person with "excellent" (E), "fair" (F), and "poor" (P), then each state has certain possibility, and we thus write the condition with a word "health condition" $(H C)$ :

$$
H C=a / E+b / F+c / P
$$

where $a, b, c \in[0,1]$ represent the possibilities of $\mathrm{E}, \mathrm{F}$, and $\mathrm{P}$, respectively. Equivalently, as in [19], the above word can also be represented as a vector $\left[\begin{array}{lll}a & b & c\end{array}\right]$. In reality, this representation leads to the formulation of fuzzy finite automata and fuzzy DESs [19,18]. It is worth indicating that fuzzy finite automata were discussed long ago by Wee [34], Santos [28,29], Lee and Zadeh [20], as well as others $[32,9,12,3,21,23,24,25]$ (the more references are referred to [21] therein). In practice, fuzzy finite automata and fuzzy languages have been used to solve meaningful problems such as intelligent interface design [5], clinical monitoring [30], neural networks $[33,4]$, and pattern recognition by DePalma and Yau (see [3,21] for the details). As well, fuzzy finite automata can be viewed as a type of formal models for computing with words $[37,36]$ when the inputs are strings of words rather than symbols. However, as the author is aware, fuzzy DESs modeled by fuzzy finite automata were not taken into account until the work of Refs. $[19,18]$ by Lin and Ying.

In [19] the authors formalized a fuzzy DES as a fuzzy finite automaton. More exactly, by means of [19] a fuzzy state is represented as a form of vector that can be viewed as a possibility distribution over the set of all considered states, that is, a word, and a fuzzy event $\tilde{\sigma}$ is equivalently represented as a matrix in which each element, say $a_{i j}$, belongs to $[0,1]$, meaning the possibility for the current state $q_{i}$ changing to state $q_{j}$ when event $\sigma$ occurs. As well, the parallel composition of fuzzy finite automata was discussed in [19]. With the appropriate formulation, Lin and Ying [19] dealt with observability and state estimation of fuzzy DESs. Furthermore, they investigated some optimal control problems. Thus, a framework of fuzzy DES theory has been primarily established in [19]. 
In supervisory control theory, how to deal with the existence of supervisor in the presence of uncontrollable events is a very fundamental issue, which was described in [1] with a number of key theorems, including Controllability Theorem and Nonblocking Controllability Theorem, and Controllability and Observability Theorem. However, these theorems in the setting of fuzzy DESs still have not been discussed. As pointed out in [19, page 409, lines 6-10], a comprehensive theory of fuzzy DESs still needs to be set up, including many fundamentally important concepts, methods, and theorems in the traditional DESs, such as controllability, observability, and optimal control. Hence, as a continuation of [19], the principal goal of this paper is to deal with controllability theorems of fuzzy DESs and some related problems.

More specifically, our main aim in the present paper is to consider a number of fundamental issues concerning supervisory control of fuzzy DESs, and the related properties. The rest of the paper is organized as follows. In Section II we review the formulation of fuzzy DESs, and define fuzzy DESs modeled by max-product and max-min automata; as well, we reformulate the parallel composition of crisp DESs, with which a parallel composition of fuzzy DESs equivalent to that in [19] is defined; notably, the computing processes in the newly defined operation may be more succinct and convenient. The focus of Section III is to deal with a number of fundamental problems of supervisory control of fuzzy DESs; we demonstrate controllability theorem and nonblocking controllability theorem of fuzzy DESs, and thus present the conditions for the existence of supervisors in fuzzy DESs; we analyze the complexity for presenting a uniform criterion to check the fuzzy controllability condition of fuzzy DESs modeled by max-product automata; in particular, if fuzzy DESs are modeled by max-min automata, then we present in detail a general method for checking whether or not the fuzzy controllability condition holds, and by means of this method we can search for all possible fuzzy states reachable from initial fuzzy state in max-min automata; furthermore, the fuzzy $n$-controllability condition is introduced for some practical problems; a number of examples are described to serve as illustrating the use of the given methods and results. Section IV investigates the properties related to supervisory control of fuzzy DESs, including the existence of supremal fuzzy controllable sublanguage and infimal prefix-closed fuzzy controllable superlanguage of a given fuzzy noncontrollable sublanguage. Finally, in Section $\mathrm{V}$ we summarize the main results obtained, and raise a number of related issues for further consideration.

Concepts and notations used in this paper will be given and explained when they first appear. 


\section{Fuzzy Discrete Event Systems}

\section{A. Language and Automaton Models of DESs}

In this subsection, we briefly review some basic concepts from formal languages and automata theory [10]. Let $\Sigma$ denote the set of events of a DES, and let $\Sigma^{*}$ denote the set of all finite length strings of events from $\Sigma$, including empty string denoted by $\epsilon$. Each sequence of events is called a string of the system, and a collection of strings is called a language. A language is regular if it is marked (or recognized) by a finite automaton, which is defined by what follows. A nondeterministic finite automaton, denoted by $G$, is a system described by $G=\left(Q, \Sigma, \delta, q_{0}, Q_{m}\right)$, where $Q$ is the finite set of states; $\Sigma$ is the finite set of events; $\delta: Q \times \Sigma \rightarrow \mathcal{P}(Q)$ is the transition function (In what follows, $\mathcal{P}(X)$ represents the power set of set $X) ; q_{0} \in Q$ is the initial state; and $Q_{m} \subseteq Q$ is called the set of marked states. Indeed, transition function $\delta$ can be naturally extended to $Q \times \Sigma^{*}$ in the following manner: For any $q \in Q$, any $s \in \Sigma^{*}$ and $\sigma \in \Sigma, \delta(q, \epsilon)=q$, and $\delta(q, s \sigma)=\delta(\delta(q, s), \sigma)$, where notably we define $\delta(A, \sigma)=\bigcup_{q \in A} \delta(q, \sigma)$ for any $A \in \mathcal{P}(Q)$. As in [17], we represent equivalently states and events by the forms of vectors and matrices, respectively. More specifically, for finite automaton $G=\left(Q, \Sigma, \delta, q_{0}, Q_{m}\right)$, where $Q=\left\{q_{1}, q_{2}, \ldots, q_{n}\right\}$, we represent $q_{i}$ by vector $\bar{s}_{i}=\left[\begin{array}{lllll}0 & \cdots & 1 & \cdots & 0\end{array}\right]$ where 1 is in the $i$ th place and the dimension equals $n$; for $\sigma \in \Sigma$, $\sigma$ is represented as a $0-1$ matrix $\left[a_{i j}\right]_{n \times n}$ where $a_{i j} \in\{0,1\}$, and $a_{i j}=1$ if and only if $q_{j} \in \delta\left(q_{i}, \sigma\right)$. Analogously, vector $\left[\begin{array}{llllllll}0 & \cdots & 1 & 0 & \cdots & 1 & \cdots & 0\end{array}\right]$ in which 1 is in the $i$ th and $j$ th places, respectively, means that the current state may be $q_{i}$ or $q_{j}$.

In order to define and better understand parallel composition of fuzzy finite automata, we reformulate the parallel composition of crisp finite automata $[1,10,11,19]$. For finite automata $G_{i}=\left(Q_{i}, \Sigma_{i}, \delta_{i}, q_{0 i}, Q_{m i}\right), i=1,2$, we reformulate the parallel composition in terms of the following fashion:

$$
G_{1} \|^{\prime} G_{2}=\left(Q_{1} \otimes Q_{2}, \Sigma_{1} \cup \Sigma_{2}, \delta_{1} \|^{\prime} \delta_{2}, q_{10} \otimes q_{20}, Q_{m 1} \otimes Q_{m 2}\right) .
$$

Here, $Q_{1} \otimes Q_{2}=\left\{q_{1} \otimes q_{2}: q_{1} \in Q_{1}, q_{2} \in Q_{2}\right\}$, and symbol " $\otimes$ " denotes tensor product that is different from the composition of fuzzy relation that means max-min operation [19]. For event $\sigma \in \Sigma_{1} \cup \Sigma_{2}$, we define the corresponding matrix of $\sigma$ in $G_{1} \|^{\prime} G_{2}$ as follows:

(i) If event $\sigma \in \Sigma_{1} \cap \Sigma_{2}$, then $\sigma=\sigma_{1} \otimes \sigma_{2}$ where $\sigma_{1}$ and $\sigma_{2}$ are the matrices of $\sigma$ in $G_{1}$ and $G_{2}$, respectively.

(ii) If event $\sigma \in \Sigma_{1} \backslash \Sigma_{2}$, then $\sigma=\sigma_{1} \otimes I_{2}$ where $\sigma_{1}$ is the matrix of $\sigma$ in $G_{1}$, and $I_{2}$ is unit 
matrix of order $\left|Q_{2}\right|$. (In this paper, $|X|$ denotes the number of all elements in set $X$.)

(iii) If event $\sigma \in \Sigma_{2} \backslash \Sigma_{1}$, then $\sigma=I_{1} \otimes \sigma_{2}$ where $\sigma_{2}$ is the matrix of $\sigma$ in $G_{2}$ and $I_{1}$ is unit matrix of order $\left|Q_{1}\right|$.

In terms of the above (i-iii) regarding the event $\sigma \in \Sigma_{1} \cup \Sigma_{2}$, we can define $\delta_{1} \|^{\prime} \delta_{2}$ as: For $q_{1} \otimes q_{2} \in Q_{1} \otimes Q_{2}, \sigma \in \Sigma_{1} \cup \Sigma_{2}$,

$$
\left(\delta_{1} \|^{\prime} \delta_{2}\right)\left(q_{1} \otimes q_{2}, \sigma\right)=\left\{\begin{array}{lll}
\left(q_{1} \otimes q_{2}\right) \times\left(\sigma_{1} \otimes \sigma_{2}\right), & \text { if } & \sigma \in \Sigma_{1} \cap \Sigma_{2}, \\
\left(q_{1} \otimes q_{2}\right) \times\left(\sigma_{1} \otimes I_{2}\right), & \text { if } & \sigma \in \Sigma_{1} \backslash \Sigma_{2}, \\
\left(q_{1} \otimes q_{2}\right) \times\left(I_{1} \otimes \sigma_{2}\right), & \text { if } & \sigma \in \Sigma_{2} \backslash \Sigma_{1},
\end{array}\right.
$$

where $\times$ is the usual product between matrices, and, as indicated above, symbol $\otimes$ denotes tensor product of matrices, that is, for $m \times n$ matrix $A=\left[a_{i j}\right]_{m \times n}$ and $k \times l$ matrix $B=\left[b_{i j}\right]_{k \times l}$, we have

$$
A \otimes B=\left[\begin{array}{lll}
a_{11} B & \cdots & a_{1 n} B \\
\vdots & \ddots & \vdots \\
a_{m 1} B & \cdots & a_{m n} B
\end{array}\right]
$$

Remark 1. One can check that for any finite automata $G_{1}$ and $G_{2}$ with event sets $\Sigma_{1}$ and $\Sigma_{2}$, respectively, the parallel composition defined above is equivalent to the usual one, that is,

$$
L\left(G_{1} \| G_{2}\right)=L\left(G_{1} \|^{\prime} G_{2}\right) \text { and } L_{m}\left(G_{1} \| G_{2}\right)=L_{m}\left(G_{1} \|^{\prime} G_{2}\right)
$$

where $G_{1} \| G_{2}$ is the usual parallel composition. In particular, when $\Sigma_{1}=\Sigma_{2}=\Sigma$, the parallel composition $\|^{\prime}$ reformulated above degenerates to the product operation $[1,10]$. We may see that defining parallel composition of finite automata by means of tensor product is more suitable to describe the composition operation with fuzzy states of each which may simultaneously belong to several crisp states with respective memberships, and with fuzzy events of each which may result in a transition between any two states with certain degree.

\section{B. Language and Automaton Models of Fuzzy DESs}

In light of Subsection A, in the setting of fuzzy DESs, a fuzzy state is naturally represented as a vector $\left[\begin{array}{llll}a_{1} & a_{2} & \cdots & a_{n}\end{array}\right]$ where $a_{i} \in[0,1]$ represents the possibility of the current state being $q_{i}$, and $n$ stands for the number of all possible crisp states. Similarly, a fuzzy event is denoted by a matrices $\left[a_{i j}\right]_{n \times n}$ as well, but here elements $a_{i j} \in[0,1]$ rather than $\{0,1\}$, and $a_{i j}$ means the possibility for the system to transit from the current state $q_{i}$ to state $q_{j}$ when event $\sigma$ 
occurs. Hence, a fuzzy finite automaton is defined as a fuzzy system $\tilde{G}=\left(\tilde{Q}, \tilde{\Sigma}, \tilde{\delta}, \tilde{q}_{0}, \tilde{Q}_{m}\right)$, where $\tilde{Q}$ is a set of some possibility distributions (fuzzy states) over crisp state set $Q ; \tilde{q}_{0}$ is the initial fuzzy state; $\tilde{Q}_{m}$ is also a set of fuzzy states over $Q$, standing for the marking states; $\tilde{\Sigma}$ is a set of fuzzy events (matrices); $\tilde{\delta}: \tilde{Q} \times \tilde{\Sigma} \rightarrow \tilde{Q}$ is a transition function which is defined by $\tilde{\delta}(\tilde{q}, \tilde{\sigma})=\tilde{q} \odot \tilde{\sigma}$ or $\tilde{q} \circ \tilde{\sigma}$ for $\tilde{q} \in \tilde{Q}$ and $\tilde{\sigma} \in \tilde{\Sigma}$, where $\odot$ and $\circ$ denote respectively max-min and max-product operations in fuzzy set theory [13]: For $n \times m$ matrix $A=\left[a_{i j}\right]$ and $m \times k$ matrix $B=\left[b_{i j}\right]$, then the elements in $n \times k$ matrixes $A \odot B=C_{1}$ and $A \circ B=C_{2}$ are respectively as $c_{i j}^{(1)}=\max _{l=1}^{m} \min \left\{a_{i l}, b_{l j}\right\}$ and $c_{i j}^{(2)}=\max _{l=1}^{m} a_{i l} \times b_{l j}$. As a complete system, we usually require that $\tilde{q}_{0} \in \tilde{Q}$ and $\tilde{Q}_{m} \subseteq \tilde{Q}$. In the sequel, a fuzzy finite automaton defined in terms of the max-min (resp. max-product) operation for its transition function is said to be max-min (resp. max-product) automaton.

The fuzzy languages generated and marked by $\tilde{G}$, denoted by $\mathcal{L}_{\tilde{G}}$ and $\mathcal{L}_{\tilde{G}, m}$, respectively, are defined as a function from $\tilde{\Sigma}^{*}\left(\tilde{\Sigma}^{*}\right.$ represents the set of all strings of fuzzy events from $\left.\tilde{\Sigma}\right)$ to $[0,1]$ as follows: For any $\tilde{\sigma}_{1} \tilde{\sigma}_{2} \cdots \tilde{\sigma}_{k} \in \tilde{\Sigma}^{*}$ where $\tilde{\sigma}_{i} \in \tilde{\Sigma}, i=1,2, \cdots, k$,

$$
\begin{gathered}
\mathcal{L}_{\tilde{G}}\left(\tilde{\sigma}_{1} \tilde{\sigma}_{2} \cdots \tilde{\sigma}_{k}\right)=\max _{i=1}^{n} \tilde{q}_{0} * \tilde{\sigma}_{1} * \tilde{\sigma}_{2} * \cdots * \tilde{\sigma}_{k} * \bar{s}_{i}^{T}, \\
\mathcal{L}_{\tilde{G}, m}\left(\tilde{\sigma}_{1} \tilde{\sigma}_{2} \cdots \tilde{\sigma}_{k}\right)=\sup _{\tilde{q} \in \tilde{Q}_{m}} \tilde{q}_{0} * \tilde{\sigma}_{1} * \tilde{\sigma}_{2} * \cdots * \tilde{\sigma}_{k} * \tilde{q}^{T},
\end{gathered}
$$

where $*$ is either max-min or max-product operation in terms of the type of $\tilde{G}$; $\bar{s}_{i}^{T}$ is the transpose of $\bar{s}_{i}$, and $\bar{s}_{i}$ is as indicated above, i.e., $\bar{s}_{i}=\left[\begin{array}{lllll}0 & \cdots & 1 & \cdots & 0\end{array}\right]$ where 1 is in the $i$ th place.

Intuitively, $\mathcal{L}_{\tilde{G}}\left(\tilde{\sigma}_{1} \tilde{\sigma}_{2} \cdots \tilde{\sigma}_{k}\right)$ represents the degree of the string of fuzzy events $\tilde{\sigma}_{1} \tilde{\sigma}_{2} \cdots \tilde{\sigma}_{k}$ being physically possible, while $\mathcal{L}_{\tilde{G}, m}\left(\tilde{\sigma}_{1} \tilde{\sigma}_{2} \cdots \tilde{\sigma}_{k}\right)$ stands for the possibility for the same string being marked (recognized) by the fuzzy automaton $\tilde{G}$. Clearly, from Eqs. (1) and (2) it follows that for any $\tilde{s} \in \tilde{\Sigma}^{*}$ and any $\tilde{\sigma} \in \tilde{\Sigma}$,

$$
\mathcal{L}_{\tilde{G}, m}(\tilde{s} \tilde{\sigma}) \leq \mathcal{L}_{\tilde{G}}(\tilde{s} \tilde{\sigma}) \leq \mathcal{L}_{\tilde{G}}(\tilde{s})
$$

which means that the degree of a string of fuzzy events being physically possible is not smaller than that of it being marked (the first inequality) and is not bigger than that of its any substring being physically possible (the second inequality).

Remark 2. The max-product automata are equivalent to the fuzzy automata defined by Lin and Ying [19], and in any max-product automaton with initial state $\tilde{q}_{0}$ and input alphabet $\tilde{\Sigma}$, the set of states $\left\{\tilde{q}_{0} \circ \tilde{s}: \tilde{s} \in \tilde{\Sigma}^{*}\right\}$ is likely infinite, and, as we will see in the 
sequel, it may result in complicated computation in realization. Therefore, we would like to consider max-min automata usually in practical applications. In reality, max-min automata are similar to the fuzzy automata defined in [30] for dealing with an application of clinical monitoring. Notably, the set of fuzzy states $\left\{\tilde{q}_{0} \odot \tilde{s}: \tilde{s} \in \tilde{\Sigma}^{*}\right\}$ in any max-min automaton is clearly finite [30].

We introduce the parallel operation of fuzzy finite automata (max-product or max-min automata). For fuzzy finite automata $\tilde{G}_{i}=\left(\tilde{Q}_{i}, \tilde{\Sigma}_{i}, \tilde{\delta}_{i}, \tilde{q}_{i 0}, \tilde{Q}_{m i}\right), i=1,2$, by means of the crisp case formulated above we can naturally define the parallel composition of $\tilde{G}_{1}$ and $\tilde{G}_{2}$ :

$$
\tilde{G}_{1} \|^{\prime} \tilde{G}_{2}=\left(\tilde{Q}_{1} \otimes \tilde{Q}_{2}, \tilde{\Sigma}_{1} \cup \tilde{\Sigma}_{2}, \tilde{\delta}_{1} \|^{\prime} \tilde{\delta}_{2}, \tilde{q}_{10} \otimes \tilde{q}_{20}, \tilde{Q}_{m 1} \otimes \tilde{Q}_{m 2}\right)
$$

where $\tilde{Q}_{1} \otimes \tilde{Q}_{2}=\left\{\tilde{q}_{1} \otimes \tilde{q}_{2}: \tilde{q}_{i}, i=1,2\right\}, \tilde{\Sigma}_{1} \cup \tilde{\Sigma}_{2}=\left\{\tilde{\sigma}: \sigma \in \Sigma_{1} \cup \Sigma_{2}\right\}$ where $\tilde{\sigma}$ is defined as follows:

(i) If $\sigma \in \Sigma_{1} \cap \Sigma_{2}$, then $\tilde{\sigma}=\tilde{\sigma}_{1} \otimes \tilde{\sigma}_{2}$ where $\tilde{\sigma}_{i}$ is the matrix of event $\sigma$ in $\tilde{G}_{i}, i=1,2$.

(ii) If event $\sigma \in \Sigma_{1} \backslash \Sigma_{2}$, then $\tilde{\sigma}=\tilde{\sigma}_{1} \otimes I_{2}$ where $\tilde{\sigma}_{1}$ is the matrix of event $\sigma$ in $\tilde{G}_{1}$ and $I_{2}$ is unit matrix of order $\left|Q_{2}\right|$.

(iii) If event $\sigma \in \Sigma_{2} \backslash \Sigma_{1}$, then $\tilde{\sigma}=I_{1} \otimes \tilde{\sigma}_{2}$ where $I_{1}$ is unit matrix of order $\left|Q_{1}\right|$ and $\tilde{\sigma}_{2}$ is equal to the matrix of $\sigma$ in $\tilde{G}_{2}$.

For any $\tilde{q}_{1} \otimes \tilde{q}_{2} \in \tilde{Q}_{1} \otimes \tilde{Q}_{2}$ and any $\tilde{\sigma} \in \tilde{\Sigma}_{1} \cup \tilde{\Sigma}_{2}$,

$$
\left(\tilde{\delta}_{1} \|^{\prime} \tilde{\delta}_{2}\right)\left(\tilde{q}_{1} \otimes \tilde{q}_{2}, \tilde{\sigma}\right)=\left(\tilde{q}_{1} \otimes \tilde{q}_{2}\right) \circ \tilde{\sigma} .
$$

Remark 3. The parallel composition $\tilde{G}_{1} \|^{\prime} \tilde{G}_{2}$ of max-product automata formulated above is equivalent to that in [19]. In [19] the parallel composition of fuzzy automata $\tilde{G}_{1}$ and $\tilde{G}_{2}$ is denoted by $\tilde{G}_{1} \| \tilde{G}_{2}=\left(\tilde{Q}_{1} \times \tilde{Q}_{2}, \tilde{\Sigma}_{1} \cup \tilde{\Sigma}_{2}, \tilde{\delta}_{1} \times \tilde{\delta}_{2},\left(\tilde{q}_{10}, \tilde{q}_{20}\right)\right)$ where every state in $\tilde{Q}_{1} \times \tilde{Q}_{2}$ is represented as the form of Cartesian product $\left(\tilde{q}_{1}, \tilde{q}_{2}\right)$ for $\tilde{q}_{1} \in \tilde{Q}_{1}$ and $\tilde{q}_{2} \in \tilde{Q}_{2}$. And it should be pointed out that the authors [19, page 411] also considered implicitly tensor product for the computing process but it was not formulated to establish the parallel composition by means of tensor product. To a certain extent, it may be more clear and convenient to describe the computing processes in terms of the parallel composition defined in this paper. An example in the following is provided to illustrate the computing.

Example 1. In fuzzy finite automata $\tilde{G}_{1}$ and $\tilde{G}_{2}$ defined above, if $\tilde{p}=\left[\begin{array}{lll}0.1 & 0.5 & 0.3\end{array}\right] \in \tilde{Q}_{1}$, $\tilde{q}=\left[\begin{array}{lll}0.2 & 0.6 & 0.1\end{array}\right] \in \tilde{Q}_{2}, \tilde{\alpha}_{1}=\left[\begin{array}{ccc}0.1 & 0.2 & 0 \\ 0.4 & 0 & 0.7 \\ 0.6 & 0.8 & 0\end{array}\right] \in \tilde{\Sigma}_{1}\left(\right.$ here $\left.\alpha_{1} \in \Sigma_{1} \backslash \Sigma_{2}\right)$, then 


$$
\begin{aligned}
& \tilde{p} \otimes \tilde{q}=\left[\begin{array}{lllllllll}
0.02 & 0.06 & 0.01 & 0.1 & 0.3 & 0.05 & 0.06 & 0.18 & 0.03
\end{array}\right], \\
& \tilde{\alpha}_{1} \otimes I_{2}=\tilde{\alpha}_{1} \otimes\left[\begin{array}{ccc}
1 & 0 & 0 \\
0 & 1 & 0 \\
0 & 0 & 1
\end{array}\right]=\left[\begin{array}{lllllllll}
0.1 & 0 & 0 & 0.2 & 0 & 0 & 0 & 0 & 0 \\
0 & 0.1 & 0 & 0 & 0.2 & 0 & 0 & 0 & 0 \\
0 & 0 & 0.1 & 0 & 0 & 0.2 & 0 & 0 & 0 \\
0.4 & 0 & 0 & 0 & 0 & 0 & 0.7 & 0 & 0 \\
0 & 0.4 & 0 & 0 & 0 & 0 & 0 & 0.7 & 0 \\
0 & 0 & 0.4 & 0 & 0 & 0 & 0 & 0 & 0.7 \\
0.6 & 0 & 0 & 0.8 & 0 & 0 & 0 & 0 & 0 \\
0 & 0.6 & 0 & 0 & 0.8 & 0 & 0 & 0 & 0 \\
0 & 0 & 0.6 & 0 & 0 & 0.8 & 0 & 0 & 0
\end{array}\right],
\end{aligned}
$$

and therefore, if $\tilde{G}_{1}$ and $\tilde{G}_{2}$ are max-min automata, then

$$
\begin{aligned}
& \tilde{\delta}_{1} \|^{\prime} \tilde{\delta}_{2}\left(\tilde{p} \otimes \tilde{q}, \tilde{\alpha}_{1}\right)=(\tilde{p} \otimes \tilde{q}) \odot\left(\tilde{\alpha}_{1} \otimes I_{2}\right) \\
& =\left[\begin{array}{lllllllll}
0.1 & 0.3 & 0.05 & 0.06 & 0.18 & 0.03 & 0.1 & 0.3 & 0.05
\end{array}\right] ;
\end{aligned}
$$

if $\tilde{G}_{1}$ and $\tilde{G}_{2}$ are max-product automata, then

$$
\begin{aligned}
& \tilde{\delta}_{1} \|^{\prime} \tilde{\delta}_{2}\left(\tilde{p} \otimes \tilde{q}, \tilde{\alpha}_{1}\right)=(\tilde{p} \otimes \tilde{q}) \circ\left(\tilde{\alpha}_{1} \otimes I_{2}\right) \\
& =[\max \{0.002,0.004,0.036\} \max \{0.006,0.12,0.108\} \max \{0.001,0.02,0.18\} \\
& \max \{0.004,0.48\} \max \{0.012,0.144\} \max \{0.002,0.24\} \quad 0.07 \quad 0.210 .35] \\
& =\left[\begin{array}{lllllllll}
0.036 & 0.12 & 0.18 & 0.48 & 0.144 & 0.24 & 0.07 & 0.21 & 0.35
\end{array}\right] \text {. }
\end{aligned}
$$

\section{Supervisory Controllability of Fuzzy DESs}

\section{A. Controllability Theorems for Fuzzy DESs}

In supervisory control, a fundamental issue is how to design a controller (or supervisor) whose task is to enable and disable the controllable events such that the resulting closed-loop system obeys some pre-specified operating rules [1]. The purpose of this subsection is to establish controllability theorem of fuzzy DESs. Again, let us recall the supervisory control problems of crisp DESs under full observation. Suppose that a DES is modeled by a finite automaton $G=\left(Q, \Sigma, \delta, q_{0}, Q_{m}\right)$ and the language $L(G)$ generated by $G$ may be interpreted as physically possible behavior. However, $L(G)$ is not satisfactory and must be modified by feedback control; modifying the behavior is to be understood as restricting the behavior to a subset of $L(G)$ that represents the "legal" behavior for the controlled system. In order to 
alter the behavior of $L(G)$, a supervisor denoted by $S$, is introduced. As a supervisor, $S$ has the capability of disabling some, but not necessarily all events of $G$. In this regard, $\Sigma$ is partitioned into two disjoint subsets $\Sigma=\Sigma_{c} \cup \Sigma_{u c}$ where $\Sigma_{c}$ is the set of controllable events that can be prevented from happening or disabled, by supervisor $S$, whereas $\Sigma_{u c}$ is the set of uncontrollable events which cannot be prevented from happening by $S$. Formally, supervisor $S$ is defined as a function:

$$
S: L(G) \rightarrow \mathcal{P}(\Sigma)
$$

It is interpreted that for each $s \in L(G), S(s) \cap\{\sigma: s \sigma \in L(G)\}$ represents the set of enabled events after the occurrence of $s$. Furthermore, it is required that for any $s \in L(G)$,

$$
\Sigma_{u c} \cap\{\sigma \in \Sigma: s \sigma \in L(G)\} \subseteq S(s)
$$

which means that after the occurrence of any physically possible string of events, the physically possible uncontrollable events are not allowed to be disabled by $S$. The condition described by Eq. (4) is called admissible for $S[1]$.

Given a DES modeled by finite automaton $G$ and admissible supervisor $S$, the resulting controlled system denoted by $S / G$ that means $S$ controlling $G$, is also a DES modeled by a language $L(S / G)$ defined recursively as follows: $\epsilon \in L(S / G)$ and

$$
s \sigma \in L(S / G) \Longleftrightarrow s \in L(S / G) \text { and } s \sigma \in L(G) \text { and } \sigma \in S(s) .
$$

The language marked by $S / G$ is defined as:

$$
L_{m}(S / G)=L(S / G) \cap L_{m}(G)
$$

The DES modeled by $L(S / G)$ is blocking if $L(S / G) \neq \operatorname{pr}\left(L_{m}(S / G)\right)$. As mentioned before, a key result in supervisory control is the controllability theorem that characterizes the existence for supervisors in the presence of uncontrollable events, and is described as follows: Suppose that a DES is modeled by finite automaton $G=\left(Q, \Sigma, \delta, q_{0}\right)$. (Here the marked set is not considered and thus left out.) Let $K \subseteq L(G)$. Then there is supervisor $S$ such that $L(S / G)=\operatorname{pr}(K)$ if and only if $\operatorname{pr}(K) \Sigma_{u c} \cap L(G) \subseteq \operatorname{pr}(K)$.

As stated before, imprecision and uncertainty play a crucial role in the field of biomedicine. For example, in practice, a single disease may show several different symptoms with respective severity degrees at every therapeutic stage. With the progress of treatment, some bad symptoms may fade away while some healthy conditions appear; also, some symptoms may weaken and the other ones possibly strength. We formally denote by $p_{1}, p_{2}, \ldots, p_{n}$ the possible main symptoms, containing healthy conditions and poor ones. If the current condition 
of a patient is with respective grades of membership $d_{i} \in[0,1]$ for $p_{i}$, denoted by $\sum_{i} d_{i}^{(1)} / p_{i}$, then a therapy (say $\tilde{\alpha}$ ) may result in another condition $\sum_{i} d_{i}^{(2)} / p_{i}$. Therefore, we will try to model such a process by means of fuzzy DESs. Furthermore, in view of human errors in medicine occurring frequently in hospitalized patients [14], we focus on dealing with supervisory control of fuzzy DESs to try to prevent medical errors such as those in diagnosis and treatment. (Fig. 1 and Fig. 2 manifest respectively the rough processes of treat without supervisor and under supervisor.)

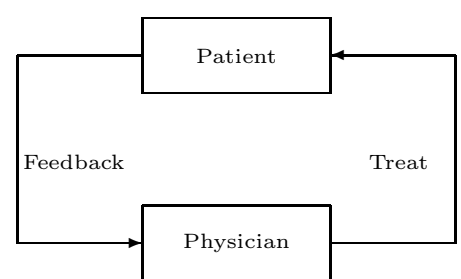

Fig. 1. Treat without supervisor.

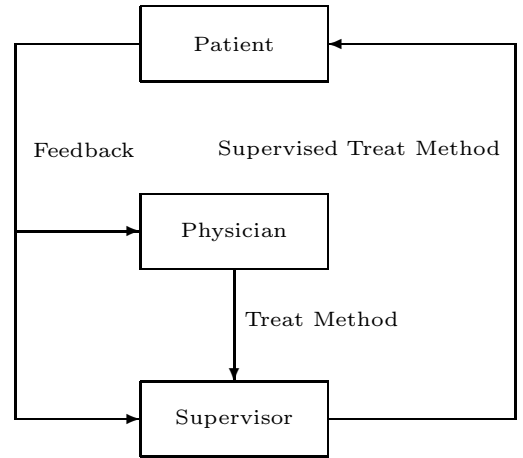

Fig. 2. Treat under supervisor.

Before giving controllability theorem in fuzzy DESs, we need further to define several related concepts in the setting of fuzzy DESs. Each event $\tilde{\sigma} \in \tilde{\Sigma}$ is associated with a degree of controllability, so, the uncontrollable set $\tilde{\Sigma}_{u c}$ and controllable set $\tilde{\Sigma}_{c}$ are two fuzzy subsets of $\tilde{\Sigma}$, i.e., $\tilde{\Sigma}_{u c}, \tilde{\Sigma}_{c} \in \mathcal{F}(\tilde{\Sigma})$ (in this paper, $\mathcal{F}(X)$ denotes the family of all fuzzy subsets of $X$ ), and satisfy: For any $\tilde{\sigma} \in \tilde{\Sigma}$,

$$
\tilde{\Sigma}_{u c}(\tilde{\sigma})+\tilde{\Sigma}_{c}(\tilde{\sigma})=1
$$

Notably, the condition described by Eq. (5) is similar to that for defining fuzzy observability by Lin and Ying [19]. For instance, in the face of deciding to treat a patient having cancer via either operation or drug therapy, either method (viewed as an fuzzy event) has certain degree to be controlled. A sublanguage of $\mathcal{L}_{\tilde{G}}$ is represented as $\tilde{K} \in \mathcal{F}\left(\tilde{\Sigma}^{*}\right)$ satisfying $\tilde{K} \subseteq \mathcal{L}_{\tilde{G}}$. In this paper, $\tilde{A} \subseteq \tilde{B}$ stands for $\tilde{A}(\tilde{\sigma}) \leq \tilde{B}(\tilde{\sigma})$ for any element $\tilde{\sigma}$ of domain. In the procedure of curing a severe patient, a single physician-in-charge will be confronted with some great challenges when making some crucial decisions, in which some mistakes also possibly happen by accident. In this regard, a supervisor (it may consist of a group of specialists) likewise plays an important role. A supervisor $\tilde{S}$ of fuzzy DES $\tilde{G}$ is defined as a function:

$$
\tilde{S}: \tilde{\Sigma}^{*} \rightarrow \mathcal{F}(\tilde{\Sigma})
$$


where for each $\tilde{s} \in \tilde{\Sigma}^{*}$ and each $\tilde{\sigma} \in \tilde{\Sigma}, \tilde{S}(\tilde{s})(\tilde{\sigma})$ represents the possibility of fuzzy event $\tilde{\sigma}$ being enabled after the occurrence of fuzzy event string $\tilde{s}$, and $\min \left\{\tilde{S}(\tilde{s})(\tilde{\sigma}), \mathcal{L}_{\tilde{G}}(\tilde{s} \tilde{\sigma})\right\}$ is interpreted to be the degree to which string $\tilde{s} \tilde{\sigma}$ is physically possible and fuzzy event $\tilde{\sigma}$ is enabled after the occurrence of fuzzy event string $\tilde{s}$. Similar to the admissibility condition Eq. (4) indicated above for crisp supervisors, $\tilde{S}$ is usually required to satisfy that for any $\tilde{s} \in \tilde{\Sigma}^{*}$ and $\tilde{\sigma} \in \tilde{\Sigma}$,

$$
\min \left\{\tilde{\Sigma}_{u c}(\tilde{\sigma}), \mathcal{L}_{\tilde{G}}(\tilde{s} \tilde{\sigma})\right\} \leq \tilde{S}(\tilde{s})(\tilde{\sigma}) .
$$

We may call this condition characterized by Eq. (6) as the fuzzy admissibility condition for supervisor $\tilde{S}$ of fuzzy DES $\tilde{G}$. To a great extent, this condition conforms to real-life control problems. For example, in a therapeutic regime for a patient having cancer, after a sequence of supervised treatments, say $\tilde{s}$, a physician and a supervisor may face the choice between operation $\tilde{\sigma}_{1}$ and drug therapy $\tilde{\sigma}_{2}$. In a way, the possibilities of $\tilde{\sigma}_{1}$ and $\tilde{\sigma}_{2}$ being controlled are small if there does not have any another therapy to be chosen at present. Likely, $\tilde{\sigma}_{1}$ or $\tilde{\sigma}_{2}$ will be adopted following $\tilde{s}$, which means that for the moment the degrees to which supervisor $\tilde{s}$ (group of specialists) can control operation $\tilde{\sigma}_{1}$ and drug therapy $\tilde{\sigma}_{2}$ are small, that is to say, $\tilde{S}(\tilde{s})\left(\tilde{\sigma}_{i}\right)(i=1,2)$ that represent the possibilities of $\tilde{\sigma}_{1}$ and $\tilde{\sigma}_{2}$ being controlled for the present are not smaller than the possibility of $\tilde{s} \tilde{\sigma}_{i}$ being implemented and $\tilde{\sigma}_{i}$ uncontrollable.

In particular, if it is only required that Eq. (6) holds true for any string $\tilde{s}$ with $|\tilde{s}| \leq n$ (in this paper, $|\tilde{s}|$ denotes the length of string $\tilde{s})$, then we call it fuzzy $n$-admissibility condition. Intuitively, the fuzzy admissibility condition Eq. (6) for supervisor $\tilde{S}$ of fuzzy DES $\tilde{G}$ means that the degree to which any fuzzy event $\tilde{\sigma} \in \tilde{\Sigma}$ following any string of fuzzy events $\tilde{s} \in$ $\tilde{\Sigma}^{*}$ is possible together with the fuzzy event $\tilde{\sigma}$ being uncontrollable is not bigger than the possibility for $\tilde{\sigma}$ being enabled after $\tilde{s}$ occurring; as such, the fuzzy $n$-admissibility condition for supervisor $\tilde{S}$ can be interpreted via restricting the length of string of fuzzy events $\tilde{s} \in \tilde{\Sigma}^{*}$ with $|\tilde{s}| \leq n$.

The fuzzy controlled system by $\tilde{S}$, denoted by $\tilde{S} / \tilde{G}$, is also a fuzzy DES and the languages $\mathcal{L}_{\tilde{S} / \tilde{G}}$ and $\mathcal{L}_{\tilde{S} / \tilde{G}, m}$ generated and marked by $\tilde{S} / \tilde{G}$ respectively are defined as follows: For any $\tilde{s} \in \tilde{\Sigma}^{*}$ and each $\tilde{\sigma} \in \tilde{\Sigma}$,

$$
\begin{aligned}
& \mathcal{L}_{\tilde{S} / \tilde{G}}(\epsilon)=1, \quad \mathcal{L}_{\tilde{S} / \tilde{G}}(\tilde{s} \tilde{\sigma})=\min \left\{\mathcal{L}_{\tilde{S} / \tilde{G}}(\tilde{s}), \mathcal{L}_{\tilde{G}}(\tilde{s} \tilde{\sigma}), \tilde{S}(\tilde{s})(\tilde{\sigma})\right\} \\
& \mathcal{L}_{\tilde{S} / \tilde{G}, m}=\mathcal{L}_{\tilde{S} / \tilde{G}} \tilde{\cap} \mathcal{L}_{\tilde{G}, m},
\end{aligned}
$$

where symbol $\tilde{\cap}$ is Zadeh fuzzy AND operator, i.e., $(\tilde{A} \tilde{\cap} \tilde{B})(x)=\min \{\tilde{A}(x), \tilde{B}(x)\}$. We give 
a notation concerning prefix-closed property in the sense of fuzzy DESs. For any $\tilde{s} \in \tilde{\Sigma}^{*}$,

$$
\operatorname{pr}(\tilde{s})=\left\{\tilde{t} \in \tilde{\Sigma}^{*}: \exists \tilde{r} \in \tilde{\Sigma}^{*}, \tilde{t} \tilde{r}=\tilde{s}\right\} .
$$

For any fuzzy language $\mathcal{L}$ over $\tilde{\Sigma}^{*}$, its prefix-closure $\operatorname{pr}(\mathcal{L}): \tilde{\Sigma}^{*} \rightarrow[0,1]$ is defined as:

$$
\operatorname{pr}(\mathcal{L})(\tilde{s})=\sup _{\tilde{s} \in \operatorname{pr}(\tilde{t})} \mathcal{L}(\tilde{t}) .
$$

So $\operatorname{pr}(\mathcal{L})(\tilde{s})$ denotes the possibility of string $\tilde{s}$ belonging to the prefix-closure of $\mathcal{L}$. By means of the formulation of the above concepts, now we can present the controllability theorem concerning fuzzy DESs.

Theorem 1. Let a fuzzy DES be modeled by fuzzy finite automaton (max-product or max-min automaton) $\tilde{G}=\left(\tilde{Q}, \tilde{\Sigma}, \tilde{\delta}, \tilde{q}_{0}\right)$. Suppose fuzzy uncontrollable subset $\tilde{\Sigma}_{u c} \in \mathcal{F}(\tilde{\Sigma})$, and fuzzy legal subset $\tilde{K} \in \mathcal{F}\left(\tilde{\Sigma}^{*}\right)$ that satisfies: $\tilde{K} \subseteq \mathcal{L}_{\tilde{G}}$, and $\tilde{K}(\epsilon)=1$. Then there exists supervisor $\tilde{S}: \tilde{\Sigma}^{*} \rightarrow \mathcal{F}(\tilde{\Sigma})$, such that $\tilde{S}$ satisfies the fuzzy admissibility condition Eq. (6) and $\mathcal{L}_{\tilde{S} / \tilde{G}}=\operatorname{pr}(\tilde{K})$ if and only if for any $\tilde{s} \in \tilde{\Sigma}^{*}$ and any $\tilde{\sigma} \in \tilde{\Sigma}$,

$$
\min \left\{\operatorname{pr}(\tilde{K})(\tilde{s}), \tilde{\Sigma}_{u c}(\tilde{\sigma}), \mathcal{L}_{\tilde{G}}(\tilde{s} \tilde{\sigma})\right\} \leq \operatorname{pr}(\tilde{K})(\tilde{s} \tilde{\sigma}),
$$

where Eq. (9) is called fuzzy controllability condition of $\tilde{K}$ with respect to $\tilde{G}$ and $\tilde{\Sigma}_{u c}$.

Intuitively, Eq. (9) means that the degree to which string $\tilde{s}$ belongs to the prefix-closure of $\tilde{K}$ and fuzzy event $\tilde{\sigma}$ following the string $\tilde{s}$ is physically possible together with $\tilde{\sigma}$ being uncontrollable is not bigger than the possibility of the string $\tilde{s} \tilde{\sigma}$ pertaining to the prefixclosure of $\tilde{K}$.

Proof of Theorem 1: First we note that $\tilde{K} \subseteq \mathcal{L}_{\tilde{G}}$ implies $\operatorname{pr}(\tilde{K}) \subseteq \mathcal{L}_{\tilde{G}}$. Indeed, for any $\tilde{s} \in \tilde{\Sigma}^{*}$, with Eq. (3) we have

$$
\operatorname{pr}(\tilde{K})(\tilde{s})=\sup _{\tilde{t} \in \tilde{\Sigma}^{*}} \tilde{K}(\tilde{s} \tilde{t}) \leq \sup _{\tilde{t} \in \tilde{\Sigma}^{*}} \mathcal{L}_{\tilde{G}}(\tilde{s} \tilde{t})=\mathcal{L}_{\tilde{G}}(\tilde{s}),
$$

which verifies this result. We begin to show that if the fuzzy controllability condition Eq. (9) holds, then there exists fuzzy supervisor $\tilde{S}$ satisfying the required conditions. We define $\tilde{S}: \tilde{\Sigma}^{*} \rightarrow \mathcal{F}(\tilde{\Sigma})$ as: For any $\tilde{s} \in \tilde{\Sigma}^{*}$ and any $\tilde{\sigma} \in \tilde{\Sigma}$,

$$
\tilde{S}(\tilde{s})(\tilde{\sigma})= \begin{cases}\min \left\{\tilde{\Sigma}_{u c}(\tilde{\sigma}), \mathcal{L}_{\tilde{G}}(\tilde{s} \tilde{\sigma})\right\}, & \text { if } \tilde{\Sigma}_{u c}(\tilde{\sigma}) \geq \operatorname{pr}(\tilde{K})(\tilde{s} \tilde{\sigma}) \\ \operatorname{pr}(\tilde{K})(\tilde{s} \tilde{\sigma}), & \text { otherwise. }\end{cases}
$$

Clearly, $\tilde{S}$ satisfies the fuzzy admissibility condition. Next our purpose is to show that for any $\tilde{s} \in \tilde{\Sigma}^{*}$,

$$
\mathcal{L}_{\tilde{S} / \tilde{G}}(\tilde{s})=\operatorname{pr}(\tilde{K})(\tilde{s}) .
$$


Proceed by induction for the length of $\tilde{s}$. If $|\tilde{s}|=0$, i.e., $\tilde{s}=\epsilon$, then $\mathcal{L}_{\tilde{S} / \tilde{G}}(\epsilon)=1=\operatorname{pr}(\tilde{K})(\epsilon)$. Suppose that Eq. (11) holds true for any $\tilde{s} \in \tilde{\Sigma}^{*}$ with $|\tilde{s}| \leq k-1$. Then our aim is to prove that Eq. (11) holds for any $\tilde{t} \in \tilde{\Sigma}^{*}$ with $|\tilde{t}|=k$. Let $\tilde{t}=\tilde{s} \tilde{\sigma}$ where $|\tilde{s}|=k-1$. Then with the assumption of induction, and the definition of $\mathcal{L}_{\tilde{S} / \tilde{G}}$, we have

$$
\begin{aligned}
\mathcal{L}_{\tilde{S} / \tilde{G}}(\tilde{s} \tilde{\sigma}) & =\min \left\{\mathcal{L}_{\tilde{S} / \tilde{G}}(\tilde{s}), \mathcal{L}_{\tilde{G}}(\tilde{s} \tilde{\sigma}), \tilde{S}(\tilde{s})(\tilde{\sigma})\right\} \\
& =\min \left\{\operatorname{pr}(\tilde{K})(\tilde{s}), \mathcal{L}_{\tilde{G}}(\tilde{s} \tilde{\sigma}), \tilde{S}(\tilde{s})(\tilde{\sigma})\right\}
\end{aligned}
$$

By means of the definition $\tilde{S}(\tilde{s})(\tilde{\sigma})$, if $\tilde{\Sigma}_{u c}(\tilde{\sigma}) \geq \operatorname{pr}(\tilde{K})(\tilde{s} \tilde{\sigma})$, then

$$
\begin{aligned}
\mathcal{L}_{\tilde{S} / \tilde{G}}(\tilde{s} \tilde{\sigma}) & =\min \left\{\operatorname{pr}(\tilde{K})(\tilde{s}), \mathcal{L}_{\tilde{G}}(\tilde{s} \tilde{\sigma}), \tilde{\Sigma}_{u c}(\tilde{\sigma})\right\} \\
& \leq \operatorname{pr}(\tilde{K})(\tilde{s} \tilde{\sigma})
\end{aligned}
$$

if $\tilde{\Sigma}_{u c}(\tilde{\sigma})<\operatorname{pr}(\tilde{K})(\tilde{s} \tilde{\sigma})$, then

$$
\begin{aligned}
\mathcal{L}_{\tilde{S} / \tilde{G}}(\tilde{s} \tilde{\sigma}) & =\min \left\{\operatorname{pr}(\tilde{K})(\tilde{s}), \mathcal{L}_{\tilde{G}}(\tilde{s} \tilde{\sigma}), \operatorname{pr}(\tilde{K})(\tilde{s} \tilde{\sigma})\right\} \\
& \leq \operatorname{pr}(\tilde{K})(\tilde{s} \tilde{\sigma})
\end{aligned}
$$

Therefore, we have shown $\mathcal{L}_{\tilde{S} / \tilde{G}}(\tilde{s} \tilde{\sigma}) \leq \operatorname{pr}(\tilde{K})(\tilde{s} \tilde{\sigma})$. On the other hand, due to $\operatorname{pr}(\tilde{K})(\tilde{s} \tilde{\sigma}) \leq$ $\operatorname{pr}(\tilde{K})(\tilde{s})$ and $\operatorname{pr}(\tilde{K})(\tilde{s} \tilde{\sigma}) \leq \mathcal{L}_{\tilde{G}}(\tilde{s} \tilde{\sigma})$, we have

$$
\begin{aligned}
\operatorname{pr}(\tilde{K})(\tilde{s} \tilde{\sigma}) & \leq \min \left\{\operatorname{pr}(\tilde{K})(\tilde{s}), \mathcal{L}_{\tilde{G}}(\tilde{s} \tilde{\sigma})\right\} \\
& =\min \left\{\mathcal{L}_{\tilde{S} / \tilde{G}}(\tilde{s}), \mathcal{L}_{\tilde{G}}(\tilde{s} \tilde{\sigma})\right\}
\end{aligned}
$$

Furthermore, if $\tilde{\Sigma}_{u c}(\tilde{\sigma}) \geq \operatorname{pr}(\tilde{K})(\tilde{s} \tilde{\sigma})$, then by combining the definition of $\tilde{S}(\tilde{s})$ with Eq. (12), we have

$$
\begin{aligned}
\operatorname{pr}(\tilde{K})(\tilde{s} \tilde{\sigma}) & \leq \min \left\{\mathcal{L}_{\tilde{S} / \tilde{G}}(\tilde{s}), \mathcal{L}_{\tilde{G}}(\tilde{s} \tilde{\sigma}), \tilde{\Sigma}_{u c}(\tilde{\sigma})\right\} \\
& =\min \left\{\mathcal{L}_{\tilde{S} / \tilde{G}}(\tilde{s}), \mathcal{L}_{\tilde{G}}(\tilde{s} \tilde{\sigma}), \tilde{S}(\tilde{s})(\tilde{\sigma})\right\} \\
& =\mathcal{L}_{\tilde{S} / \tilde{G}}(\tilde{s} \tilde{\sigma})
\end{aligned}
$$

if $\tilde{\Sigma}_{u c}(\tilde{\sigma})<\operatorname{pr}(\tilde{K})(\tilde{s} \tilde{\sigma})$, then with the definition of $\tilde{S}(\tilde{s})$ we have $\tilde{S}(\tilde{s})(\tilde{\sigma})=\operatorname{pr}(\tilde{K})(\tilde{s} \tilde{\sigma})$, and by Eq. (12) we obtain that

$$
\begin{aligned}
\operatorname{pr}(\tilde{K})(\tilde{s} \tilde{\sigma}) & \leq \min \left\{\mathcal{L}_{\tilde{S} / \tilde{G}}(\tilde{s}), \mathcal{L}_{\tilde{G}}(\tilde{s} \tilde{\sigma}), \tilde{S}(\tilde{s})(\tilde{\sigma})\right\} \\
& =\mathcal{L}_{\tilde{S} / \tilde{G}}(\tilde{s} \tilde{\sigma}) .
\end{aligned}
$$

Therefore we have verified that $\operatorname{pr}(\tilde{K})(\tilde{s} \tilde{\sigma})=\mathcal{L}_{\tilde{S} / \tilde{G}}(\tilde{s} \tilde{\sigma})$ holds for any $\tilde{s} \in \tilde{\Sigma}^{*}$ and $\tilde{\sigma} \in \tilde{\Sigma}$ with $|\tilde{s}|=k-1$, and the proof of sufficiency is completed. 
The remainder is to consider the proof of necessity. If $\mathcal{L}_{\tilde{S} / \tilde{G}}=\operatorname{pr}(\tilde{K})$ holds, then for any $\tilde{s} \in \tilde{\Sigma}^{*}$ and $\tilde{\sigma} \in \tilde{\Sigma}$, with the fuzzy admissibility condition Eq. (6) of $\tilde{S}$ we have

$$
\begin{aligned}
& \min \left\{\operatorname{pr}(\tilde{K})(\tilde{s}), \tilde{\Sigma}_{u c}(\tilde{\sigma}), \mathcal{L}_{\tilde{G}}(\tilde{s} \tilde{\sigma})\right\} \\
\leq & \min \left\{\mathcal{L}_{\tilde{S} / \tilde{G}}(\tilde{s}), \tilde{S}(\tilde{s})(\tilde{\sigma}), \mathcal{L}_{\tilde{G}}(\tilde{s} \tilde{\sigma})\right\} \\
= & \mathcal{L}_{\tilde{S} / \tilde{G}}(\tilde{s} \tilde{\sigma}) \\
= & \operatorname{pr}(\tilde{K})(\tilde{s} \tilde{\sigma}) .
\end{aligned}
$$

This completes the proof of necessity and the theorem has therefore been proved.

Remark 4. Theorem 1 together with its proof presents a method for designing a supervisor of a fuzzy DES such that the deriving fuzzy system obeys the pre-specified "legal" behavior for the fuzzy controlled system, which may apply to fuzzy DESs such as modeling a patient's health condition and traffic systems. In particular, from the above proof we see that in Theorem 1 the fuzzy finite automaton can be of max-product or max-min. We will see that when fuzzy DES is modeled by max-product automaton, it is difficult to check the fuzzy controllability condition Eq. (9) with a general criterion, whereas if max-min automata are used to model fuzzy DESs, then we can present a general computing process in detail for testing the fuzzy controllability condition Eq. (9).

As an aspect of application, we can utilize Theorem 1 to cope with some realistic control problems with finite length specifications. In this regard, from Theorem 1 it follows readily the following Corollary 1. Before giving this corollary, we introduce two notations: For any two fuzzy languages $\tilde{A}$ and $\tilde{B}$ over set $\tilde{\Sigma}$ of fuzzy events, $\tilde{A} \subseteq \subseteq_{n} \tilde{B}$ and $\tilde{A}={ }_{n} \tilde{B}$ mean respectively that for any string $\tilde{s} \in \tilde{\Sigma}^{*}$ with $|\tilde{s}| \leq n, \tilde{A}(\tilde{s}) \leq \tilde{B}(\tilde{s})$ and $\tilde{A}(\tilde{s})=\tilde{B}(\tilde{s})$.

Corollary 1. Let a fuzzy DES be modeled by fuzzy finite automaton (max-product or max$\min ) \tilde{G}=\left(\tilde{Q}, \tilde{\Sigma}, \tilde{\delta}, \tilde{q}_{0}\right)$. Let $n$ be any given positive integer. Suppose fuzzy uncontrollable subset $\tilde{\Sigma}_{u c} \in \mathcal{F}(\tilde{\Sigma})$, and fuzzy legal subset $\tilde{K} \in \mathcal{F}\left(\tilde{\Sigma}^{*}\right)$ that satisfies: $\tilde{K} \subseteq_{n} \mathcal{L}_{\tilde{G}}$, and $\tilde{K}(\epsilon)=1$. Then there exists supervisor $\tilde{S}: \tilde{\Sigma}^{*} \rightarrow \mathcal{F}(\tilde{\Sigma})$, such that $\tilde{S}$ satisfies the fuzzy $n$-admissibility condition Eq. (6) and $\mathcal{L}_{\tilde{S} / \tilde{G}}={ }_{n} \operatorname{pr}(\tilde{K})$ if and only if for any $\tilde{s} \in \tilde{\Sigma}^{*}$ with $|\tilde{s}| \leq n$ and any $\tilde{\sigma} \in \tilde{\Sigma}$,

$$
\min \left\{\operatorname{pr}(\tilde{K})(\tilde{s}), \tilde{\Sigma}_{u c}(\tilde{\sigma}), \mathcal{L}_{\tilde{G}}(\tilde{s} \tilde{\sigma})\right\} \leq \operatorname{pr}(\tilde{K})(\tilde{s} \tilde{\sigma}),
$$

where Eq. (13) is called fuzzy n-controllability condition of $\tilde{K}$ with respect to $\tilde{G}$ and $\tilde{\Sigma}_{u c}$.

Proof: Exactly similar to Theorem 1 by restricting the length of $\tilde{s}$ with $|\tilde{s}| \leq n$.

Remark 5. If for every fuzzy event $\tilde{\sigma} \in \tilde{\Sigma}$ and every string $\tilde{s} \in \tilde{\Sigma}^{*}$, we view the total 
computation for Eq. (13) as a step, then with Eq. (13) it is possible to check whether or not fuzzy $n$-controllability condition holds with most number of computing steps $(1+$ $\left.|\tilde{\Sigma}|+2^{|\tilde{\Sigma}|}+\cdots+n^{|\tilde{\Sigma}|}\right)|\tilde{\Sigma}|$, where $|\tilde{\Sigma}|$ is the number of fuzzy events. Therefore the worst-case computational complexity for this test is exponential, and it should be worth considering to reduce the computation procedure.

\section{B. Realization of Supervisors for Fuzzy DESs and Some Examples}

From Theorem 1 it is seen that the existence of supervisor $\tilde{S}$ is closely related to the fuzzy controllability condition of $\tilde{K}$ defined by Eq. (9), so it is very important to consider how to test such a condition. Nevertheless, in fuzzy DESs modeled by max-product automata, the set of fuzzy states $\left\{\tilde{q}_{0} \circ \tilde{s}: \tilde{s} \in \tilde{\Sigma}^{*}\right\}$ is likely infinite. Along with the further analysis in the following (Case 1), it is quite complicated to present a uniform method to check the fuzzy controllability condition for this case. But when the fuzzy DESs modeled by max-min automata, we can give a general computing flow for testing the condition (Case 2). The two cases are dealt with as follows, and we focus on the second case.

Case 1. Fuzzy DESs modeled by max-product automata.

In fuzzy DES modeled by max-product automaton $\tilde{G}=\left(\tilde{Q}, \tilde{\Sigma}, \tilde{\delta}, \tilde{q}_{0}\right)$, in which the dimensionality of $\tilde{Q}$ is $n$ (i.e., $Q$ has $n$ crisp states), for a given fuzzy subset $\tilde{K} \subseteq \mathcal{L}_{\tilde{G}}$ of control specifications, by means of Theorem 1 we should decide the existence of supervisor $\tilde{S}$. As in crisp DESs [1], we may assume $\operatorname{pr}(\tilde{K})$ to be a fuzzy language generated by a maxproduct automaton $\tilde{H}=\left(\tilde{R}, \tilde{\Sigma}, \tilde{\gamma}, \tilde{p_{0}}\right)$ with dimensionality $m$ of $\tilde{R}$. Then we have that for any $\tilde{s}=\tilde{\sigma}_{1} \tilde{\sigma}_{2} \cdots \tilde{\sigma}_{k} \in \tilde{\Sigma}^{*}$ where $\tilde{\sigma}_{i} \in \tilde{\Sigma}, i=1,2, \cdots, k$,

$$
\begin{gathered}
\operatorname{pr}(\tilde{K})(\tilde{s})=\mathcal{L}_{\tilde{H}}(\tilde{s})=\max _{i=1}^{m} \tilde{p}_{0} \circ \tilde{\sigma}_{1} \circ \tilde{\sigma}_{2} \circ \cdots \circ \tilde{\sigma}_{k} \circ \bar{s}_{i}^{T}, \\
\operatorname{pr}(\tilde{K})(\tilde{s} \tilde{\sigma})=\mathcal{L}_{\tilde{H}}(\tilde{s} \tilde{\sigma})=\max _{i=1}^{m} \tilde{p}_{0} \circ \tilde{\sigma}_{1} \circ \tilde{\sigma}_{2} \circ \cdots \circ \tilde{\sigma}_{k} \circ \tilde{\sigma} \circ \bar{s}_{i}^{T}, \\
\mathcal{L}_{\tilde{G}}(\tilde{s} \tilde{\sigma})=\max _{i=1}^{m} \tilde{q_{0}} \circ \tilde{\sigma}_{1} \circ \tilde{\sigma}_{2} \circ \cdots \circ \tilde{\sigma}_{k} \circ \tilde{\sigma} \circ \bar{s}_{i}^{T},
\end{gathered}
$$

where $\bar{s}_{i}^{T}(i=1,2, \ldots, m)$ and $\bar{s}_{j}^{T}(j=1,2, \ldots, m)$, as indicated above, are respectively the crisp states of $\tilde{H}$ and $\tilde{G}$. If the sets of fuzzy states $\left\{\tilde{p_{0}} \circ \tilde{s}: \tilde{s} \in \tilde{\Sigma}^{*}\right\}$ and $\left\{\tilde{q_{0}} \circ \tilde{s}: \tilde{s} \in \tilde{\Sigma}^{*}\right\}$ were finite, then by virtue of Eqs. (14-16) we can check the fuzzy controllability condition described above by Eq. (9) within finite steps. But unfortunately it is perhaps not so. From Eqs. (14) and (15) it follows that $\operatorname{pr}(\tilde{K})(\tilde{s})$ and $\operatorname{pr}(\tilde{K})(\tilde{s} \tilde{\sigma})$ may be arbitrarily small in case of the length of $\tilde{s}$ big enough, since those elements in fuzzy events (matrices) are usually smaller 
than 1. On the other hand, $\operatorname{pr}(\tilde{K})(\tilde{s} \tilde{\sigma}) \leq \mathcal{L}_{\tilde{G}}(\tilde{s} \tilde{\sigma})$ is prerequisite, and $\operatorname{pr}(\tilde{K})(\tilde{s} \tilde{\sigma}) \leq \operatorname{pr}(\tilde{K})(\tilde{s})$ always holds, that is, we always have

$$
\min \left\{\operatorname{pr}(\tilde{K})(\tilde{s} \tilde{\sigma}), \tilde{\Sigma}_{u c}(\tilde{\sigma})\right\} \leq \min \left\{\operatorname{pr}(\tilde{K})(\tilde{s}), \tilde{\Sigma}_{u c}(\tilde{\sigma}), \mathcal{L}_{\tilde{G}}(\tilde{s} \tilde{\sigma})\right\}
$$

however, $\tilde{\Sigma}_{u c}(\tilde{\sigma})$ is certain for each fuzzy event $\tilde{\sigma}$, and, as just indicated, $\operatorname{pr}(\tilde{K})(\tilde{s})$ and $\operatorname{pr}(\tilde{K})(\tilde{s} \tilde{\sigma})$ tend likely to zero when $|\tilde{s}|$ is big enough, and it thus follows from Eq. (17) that

$$
\operatorname{pr}(\tilde{K})(\tilde{s} \tilde{\sigma}) \leq \min \left\{\operatorname{pr}(\tilde{K})(\tilde{s}), \tilde{\Sigma}_{u c}(\tilde{\sigma}), \mathcal{L}_{\tilde{G}}(\tilde{s} \tilde{\sigma})\right\}
$$

which implies that to guarantee the fuzzy controllability condition Eq. (9), it is required that as the length of string $\tilde{s}$ becomes big enough,

$$
\operatorname{pr}(\tilde{K})(\tilde{s} \tilde{\sigma})=\min \left\{\operatorname{pr}(\tilde{K})(\tilde{s}), \mathcal{L}_{\tilde{G}}(\tilde{s} \tilde{\sigma})\right\}
$$

Clearly, Eq. (18) is a strict condition on $\tilde{K}$, and, therefore, to ensure the fuzzy controllability condition Eq. (9), some restrictions imposed upon $\tilde{K}$ are necessary. For example,

$$
\tilde{K}(\tilde{s} \tilde{\sigma}) \geq \min \left\{\tilde{\Sigma}_{u c}(\tilde{\sigma}), \mathcal{L}_{\tilde{G}}(\tilde{s} \tilde{\sigma})\right\}
$$

is a sufficient condition to result in Eq. (9); or when the support set of $\tilde{K}$ is finite, we can check whether or not Eq. (9) holds.

Case 2. Fuzzy DESs modeled by max-min automata.

Let fuzzy DES be modeled by max-min automaton $\tilde{G}=\left(\tilde{Q}, \tilde{\Sigma}, \tilde{\delta}, \tilde{q}_{0}\right)$, in which the dimensionality of $\tilde{Q}$ is $n$. We first give a computing tree for deriving the set of all fuzzy states reachable from the initial state $\tilde{q}_{0}$, and the sets of strings respectively corresponding to each accessible fuzzy state are also obtained. Assume that $\tilde{\Sigma}=\left\{\tilde{\alpha}_{1}, \tilde{\alpha}_{2}, \ldots, \tilde{\alpha}_{n}\right\}$. A basic idea is based on that $(\mathrm{i}) \tilde{q}_{0} \odot \tilde{s}=\tilde{q}_{0} \odot \tilde{s} \odot\left(\tilde{s}_{1}\right)^{n}$ for any $n \geq 0$ if $\tilde{q}_{0} \odot \tilde{s}=\tilde{q}_{0} \odot \tilde{s} \odot \tilde{s}_{1}$ for $\tilde{s}_{1} \in \tilde{\Sigma}^{*}$, where $\left(\tilde{s}_{1}\right)^{n}$ denotes the $\odot$ product of $n$ 's $\tilde{s}_{1}$, and (ii) the set of fuzzy states $\left\{\tilde{q}_{0} \odot \tilde{s}: \tilde{s} \in \tilde{\Sigma}^{*}\right\}$ is always finite since $\tilde{\Sigma}$ is finite. For the sake of simplicity, we present the computing tree for $\tilde{\Sigma}=\left\{\tilde{\alpha}_{1}, \tilde{\alpha}_{2}\right\}$ of two fuzzy events via Fig. 3, and the case of more than two fuzzy events is analogous. 


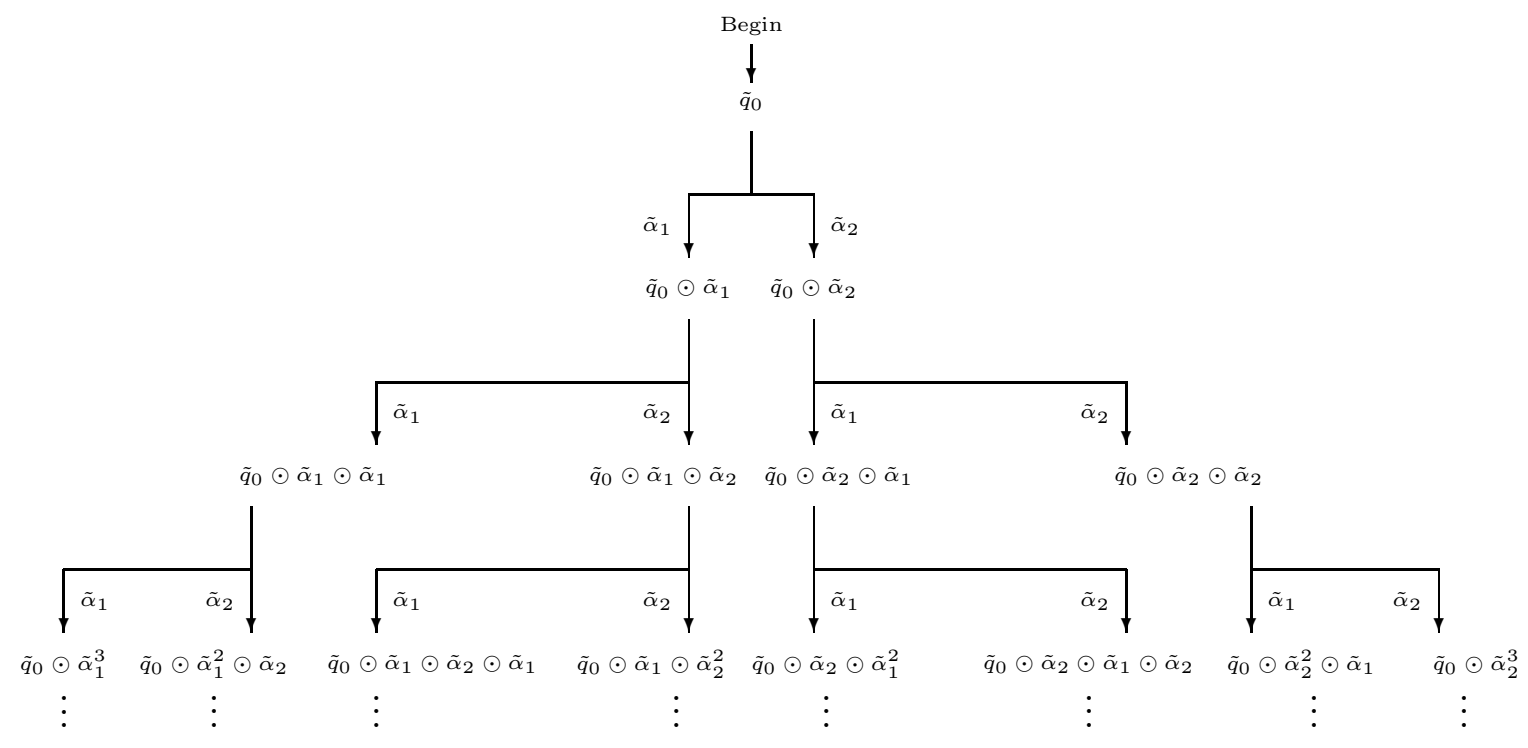

Fig. 3. A computing tree for deciding the all different fuzzy states reachable from $\tilde{q}_{0}$.

In this computing tree, the initial fuzzy state $\tilde{q}_{0}$ is its root; each vertex, say $\tilde{q}_{0} \odot \tilde{s}$, may produce $n$ 's sons $\tilde{q}_{0} \odot \tilde{s} \odot \tilde{s}_{1}, \tilde{q}_{0} \odot \tilde{s} \odot \tilde{s}_{2}, \ldots, \tilde{q}_{0} \odot \tilde{s} \odot \tilde{s}_{1}$. However, if $\tilde{q}_{0} \odot \tilde{s}$ equals some its father, then $\tilde{q}_{0} \odot \tilde{s}$ is a leaf, that is marked by a underline. The computing ends with a leaf at the end of each branch.

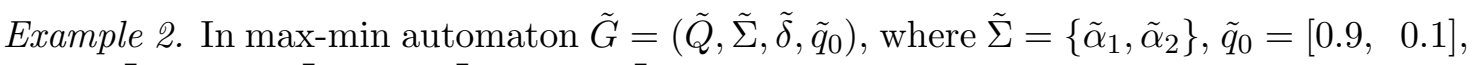
and $\tilde{\alpha}_{1}=\left[\begin{array}{cc}0.4 & 0.8 \\ 0.2 & 0.2\end{array}\right], \tilde{\alpha}_{2}=\left[\begin{array}{cc}0.4 & 0.2 \\ 0.8 & 0.5\end{array}\right]$. Then we have the following computing tree, in which each leaf is underlined. 


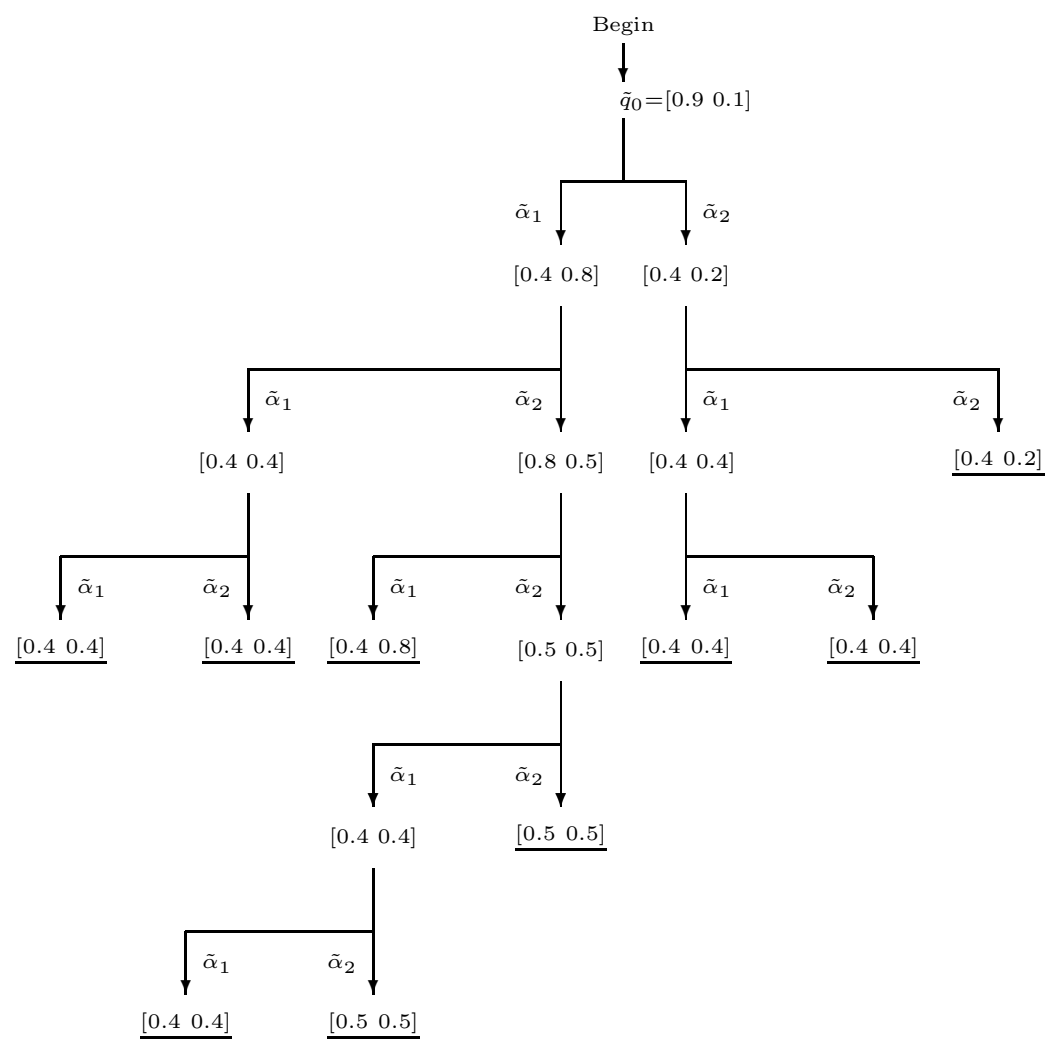

Fig. 4. A computing tree for deciding the all different fuzzy states reachable from $\tilde{q}_{0}=\left[\begin{array}{lll}0.9 & 0.1\end{array}\right]$.

From this computing tree (Fig. 4) it follows that in $\tilde{G}$ there are only six different fuzzy states reachable from $\tilde{q}_{0}=[0.90 .1]$, which are listed in TABLE I as follows.

TABLE I

Six different fuzzy states reachable from $\tilde{q}_{0}$.

\begin{tabular}{|c|c|c|c|}
\hline$\tilde{s}$ & $\tilde{q}_{0} \odot \tilde{s}$ & $\tilde{s}$ & $\tilde{q}_{0} \odot \tilde{s}$ \\
\hline$\tilde{\epsilon}$ & {$\left[\begin{array}{ll}0.9 & 0.1\end{array}\right]$} & $\tilde{\alpha}_{1} \tilde{\alpha}_{1}$ & {$\left[\begin{array}{ll}0.4 & 0.4\end{array}\right]$} \\
\hline$\tilde{\alpha}_{1}$ & {$\left[\begin{array}{ll}0.4 & 0.8\end{array}\right]$} & $\tilde{\alpha}_{1} \tilde{\alpha}_{2}$ & {$\left[\begin{array}{ll}0.8 & 0.5\end{array}\right]$} \\
\hline$\tilde{\alpha}_{2}$ & {$\left[\begin{array}{ll}0.4 & 0.2\end{array}\right]$} & $\tilde{\alpha}_{1} \tilde{\alpha}_{2} \tilde{\alpha}_{2}$ & {$\left[\begin{array}{ll}0.5 & 0.5\end{array}\right]$} \\
\hline
\end{tabular}

Also, the corresponding sets of strings to each fuzzy states can be obtained respectively as follows: $C\left(\tilde{q}_{0}\right)=\{\epsilon\} ; C\left(\tilde{q}_{0} \odot \tilde{\alpha}_{1}\right)=\left\{\tilde{\alpha}_{1}\left(\tilde{\alpha}_{2} \tilde{\alpha}_{1}\right)^{n}: n \geq 0\right\} ; C\left(\tilde{q}_{0} \odot \tilde{\alpha}_{2}\right)=\left\{\tilde{\alpha}_{2}^{n}: n \geq 1\right\}$;

$$
\begin{aligned}
C\left(\tilde{q}_{0} \odot \tilde{\alpha}_{1}^{2}\right)= & \left\{\tilde{\alpha}_{1}^{2} \tilde{\alpha}_{1}^{n}: n \geq 0\right\} \cup\left\{\tilde{\alpha}_{1}^{2} \tilde{\alpha}_{2}^{n}: n \geq 0\right\} \\
& \cup\left\{\tilde{\alpha}_{1} \tilde{\alpha}_{2} \tilde{\alpha}_{2} \tilde{\alpha}_{1}^{n}: n \geq 1\right\} \cup\left\{\tilde{\alpha}_{1} \tilde{\alpha}_{2} \tilde{\alpha}_{2} \tilde{\alpha}_{1} \tilde{\alpha}_{2}^{n}: n \geq 0\right\} \\
& \cup\left\{\tilde{\alpha}_{2} \tilde{\alpha}_{1}^{n}: n \geq 1\right\} \cup\left\{\tilde{\alpha}_{2} \tilde{\alpha}_{1} \tilde{\alpha}_{2}^{n}: n \geq 0\right\} ;
\end{aligned}
$$

$C\left(\tilde{q}_{0} \odot \tilde{\alpha}_{1} \odot \tilde{\alpha}_{2}\right)=\left\{\left(\tilde{\alpha}_{1} \tilde{\alpha}_{2}\right)^{n}: n \geq 1\right\} ;$ and $C\left(\tilde{q}_{0} \odot \tilde{\alpha}_{1} \odot \tilde{\alpha}_{2}^{2}\right)=\left\{\tilde{\alpha}_{1} \tilde{\alpha}_{2} \tilde{\alpha}_{2}^{n}: n \geq 1\right\}$. Here, $C(\tilde{q})$ denotes the set $\left\{\tilde{s} \in \tilde{\Sigma}^{*}: \tilde{\delta}\left(\tilde{q}_{0}, \tilde{s}\right)=\tilde{q}_{0} \odot \tilde{s}=\tilde{q}\right\}$.

For two max-min automata $\tilde{G}_{i}=\left(\tilde{Q}_{i}, \tilde{\Sigma}_{i}, \tilde{\delta}_{i}, \tilde{q}_{i 0}\right)(i=1,2)$ with the same set of fuzzy events $\tilde{\Sigma}_{1}=\tilde{\Sigma}_{2}=\tilde{\Sigma}=\left\{\tilde{\sigma}_{1}, \tilde{\sigma}_{2}, \ldots, \tilde{\sigma}_{n}\right\}$, our purpose is to search for the all different pairs of fuzzy states reachable from the initial fuzzy state pair $\left(\tilde{q}_{10}, \tilde{q}_{20}\right)$, that is, $\left\{\left(\tilde{q}_{10} \odot \tilde{s}, \tilde{q}_{20} \odot \tilde{s}\right)\right.$ : $\left.\tilde{s} \in \tilde{\Sigma}^{*}\right\}$. The method is similar to the case of single max-min automaton presented above, 
that is carried out by a computing tree. In the computing tree, the root is labeled with pair $\left(\tilde{q}_{10}, \tilde{q}_{20}\right)$, and each vertex, say $\left(\tilde{q}_{10} \odot \tilde{s}, \tilde{q}_{20} \odot \tilde{s}\right)$ for $\tilde{s} \in \tilde{\Sigma}^{*}$, may produce $n$ 's sons, i.e., $\left(\tilde{q}_{10} \odot \tilde{s} \odot \tilde{\sigma}_{i}, \tilde{q}_{20} \odot \tilde{s} \odot \tilde{\sigma}_{i}\right), i=1,2, \ldots, n$. But if a pair $\left(\tilde{q}_{10} \odot \tilde{s} \odot \tilde{\sigma}_{i}, \tilde{q}_{20} \odot \tilde{s} \odot \tilde{\sigma}_{i}\right)$ is the same as one of its father, then this pair will be treated as a leaf, that is marked with a underline. Such a computing tree is depicted by Fig. 5 as follows. Since the set of all pairs of fuzzy states is finite due to the finiteness of $\tilde{\Sigma}$, the computing tree ends with a leaf at the end of each branch.

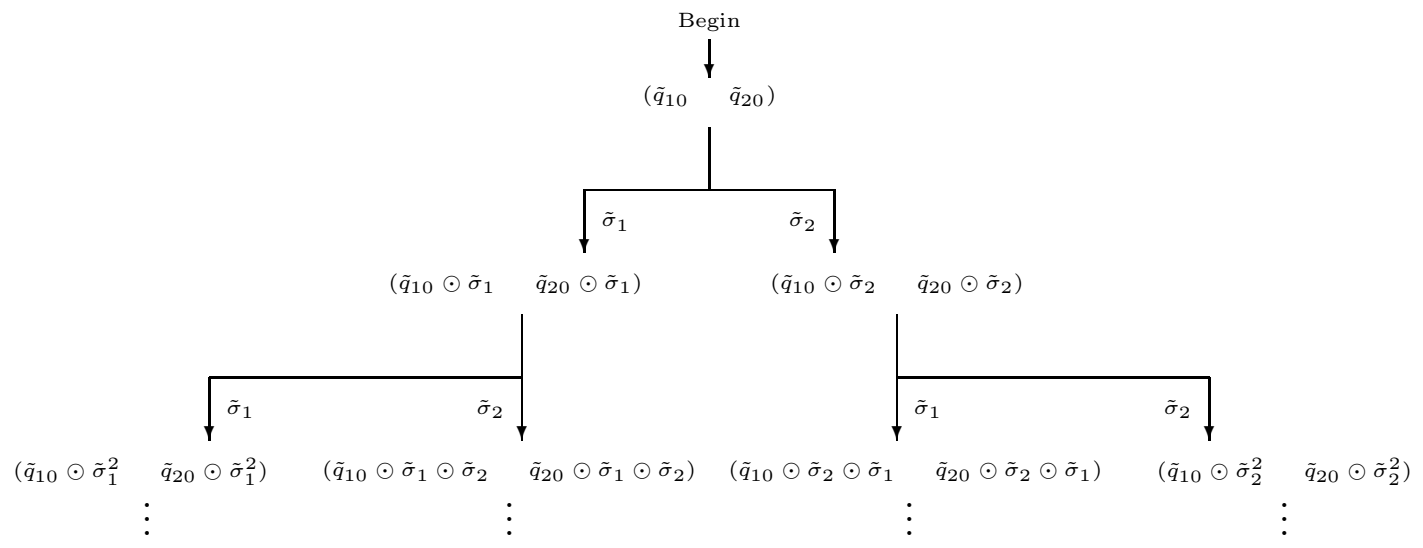

Fig. 5. The computing tree deciding the all different pairs of fuzzy states reachable from the initial fuzzy state pair $\left(\tilde{q}_{10}, \tilde{q}_{20}\right)$.

Example 3. For max-min automata $\tilde{G}=\left(\tilde{Q}, \tilde{\Sigma}, \tilde{\delta}, \tilde{q}_{0}\right)$ as Example 2 , and $\tilde{H}=\left(\tilde{Q}_{1}, \tilde{\Sigma}, \tilde{\gamma}, \tilde{p}_{0}\right)$, where $\tilde{p}_{0}=\left[\begin{array}{ll}0.8 & 0.2\end{array}\right], \tilde{\Sigma}=\left\{\tilde{\alpha}_{1}, \tilde{\alpha}_{2}\right\}$ with $\tilde{\alpha}_{1}=\left[\begin{array}{cc}0.2 & 0.8 \\ 0.2 & 0.2\end{array}\right], \tilde{\alpha}_{2}=\left[\begin{array}{cc}0.2 & 0.2 \\ 0.8 & 0.5\end{array}\right]$, then from the above computing tree Fig. 5 we can derive the computing tree Fig. 6 for deciding the set of all different fuzzy state pairs reachable from $\left(\tilde{q}_{0}, \tilde{p}_{0}\right)$ as follows: 


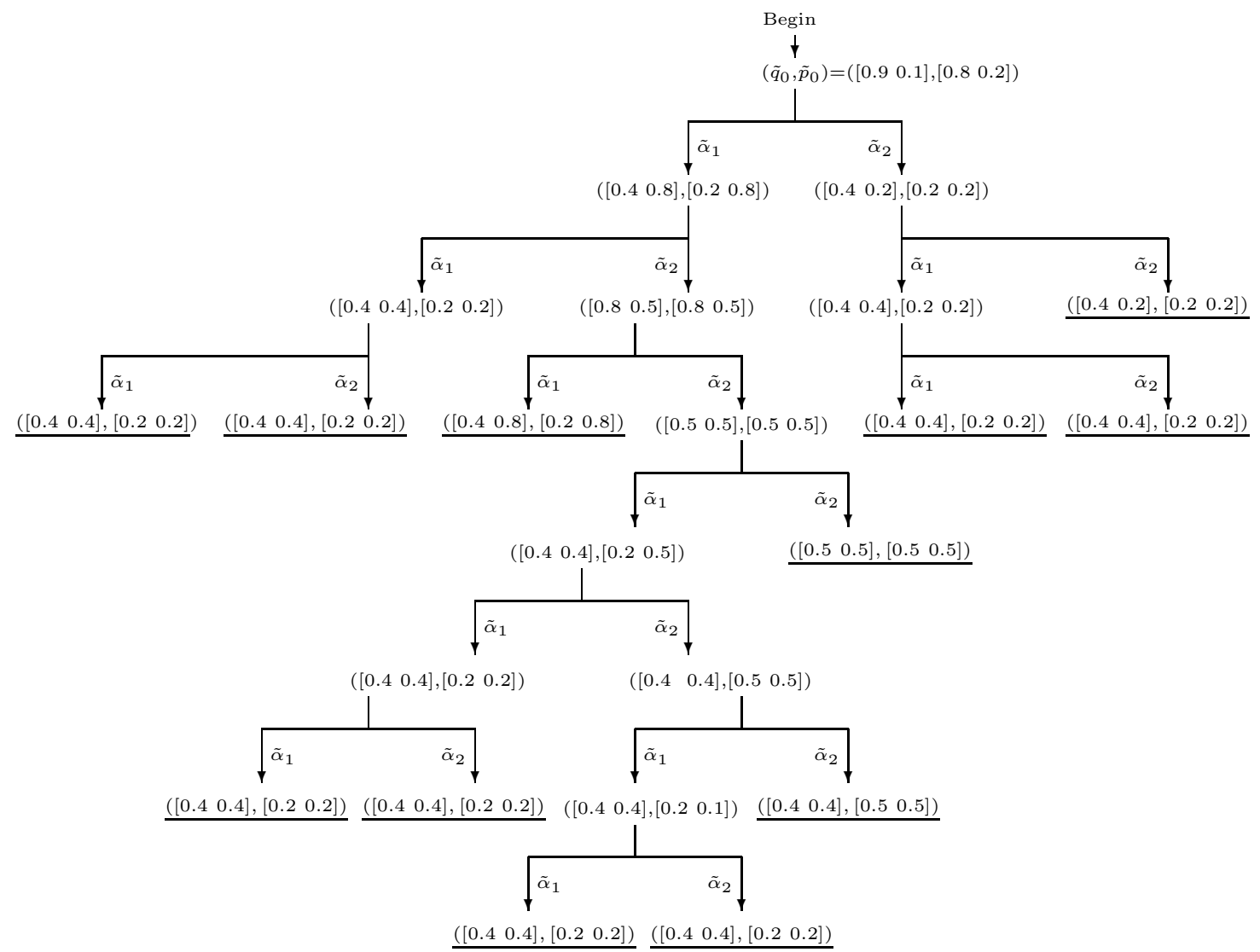

Fig. 6. The computing tree deciding the all different pairs of fuzzy states reachable from the initial fuzzy state pair $\left(\tilde{q}_{0}, \tilde{p}_{0}\right)=\left(\left[\begin{array}{ll}0.9 & 0.1\end{array}\right],\left[\begin{array}{ll}0.8 & 0.2\end{array}\right]\right)$.

On the basis of the above discussion, if $\operatorname{pr}(\tilde{K})$ is generated by a max-min automaton $\tilde{H}=$ $\left(\tilde{Q}_{1}, \tilde{\Sigma}, \tilde{\gamma}, \tilde{p}_{0}\right)$, we can test the fuzzy controllability condition Eq. (9) within finite steps by means of TABLE II, where

$$
\begin{aligned}
& L(\tilde{G}, \tilde{H}, \tilde{s}, \tilde{\sigma})=\min \left\{\operatorname{pr}(\tilde{K})(\tilde{s}), \tilde{\Sigma}_{u c}(\tilde{\sigma}), \mathcal{L}_{\tilde{G}}(\tilde{s} \tilde{\sigma})\right\}, \\
& \operatorname{pr}(\tilde{K})(\tilde{s})=\mathcal{L}_{\tilde{H}}(\tilde{s})=\left[\tilde{p}_{0} \odot \tilde{s}\right], \\
& \mathcal{L}_{\tilde{G}}(\tilde{s} \tilde{\sigma})=\left[\tilde{q}_{0} \odot \tilde{s} \odot \tilde{\sigma}\right] .
\end{aligned}
$$

Here the set of all different fuzzy state pairs is assumed to be

$$
\left\{\left(\tilde{q}_{0} \odot \tilde{s}, \tilde{p}_{0} \odot \tilde{s}\right): \tilde{s}_{1}, \tilde{s}_{2}, \ldots, \tilde{s}_{m} \in \tilde{\Sigma}^{*}\right\}
$$

In TABLE II, $L(\tilde{G}, \tilde{H}, \tilde{s}, \tilde{\sigma}) \leq\left[\tilde{p}_{0} \odot \tilde{s} \odot \tilde{\sigma}\right]$ is exactly the fuzzy controllability condition Eq. (9); if all elements in the rightmost column are "T", then the fuzzy controllability condition Eq. (9) holds true; otherwise, it does not hold. 
TABLE II. Testing the fuzzy controllability condition Eq. (9) in terms of whether or not the all elements in the rightmost column are "T (True)"

\begin{tabular}{|c|c|c|c|c|c|c|c|}
\hline$\tilde{s}$ & $\tilde{\sigma}$ & {$\left[\tilde{p}_{0} \odot \tilde{s}\right]$} & {$\left[\tilde{q}_{0} \odot \tilde{s} \odot \tilde{\sigma}\right]$} & $\tilde{\Sigma}_{u c}(\tilde{\sigma})$ & $L(\tilde{G}, \tilde{H}, \tilde{s}, \tilde{\sigma})$ & {$\left[\tilde{p}_{0} \odot \tilde{s} \odot \tilde{\sigma}\right]$} & $L(\tilde{G}, \tilde{H}, \tilde{s}, \tilde{\sigma}) \leq\left[\tilde{p}_{0} \odot \tilde{s} \odot \tilde{\sigma}\right]$ \\
\hline \multirow[t]{3}{*}{$\tilde{s}_{1}$} & $\tilde{\sigma}_{1}$ & & & & & & $\mathrm{~T}$ (True) or F (False) \\
\hline & & & & & & & $\mathrm{T}$ (True) or F (False) \\
\hline & $\tilde{\sigma}_{n}$ & & & & & & $\mathrm{~T}$ (True) or F (False) \\
\hline \multirow[t]{3}{*}{$\tilde{s}_{2}$} & $\tilde{\sigma}_{1}$ & & & & & & $\mathrm{~T}$ (True) or F (False) \\
\hline & $\vdots$ & & & & & & $\mathrm{T}$ (True) or F (False) \\
\hline & $\tilde{\sigma}_{n}$ & & & & & & $\mathrm{~T}$ (True) or F (False) \\
\hline$\vdots$ & $\vdots$ & & & & & & $\mathrm{T}$ (True) or F (False) \\
\hline \multirow[t]{3}{*}{$\tilde{s}_{m}$} & $\tilde{\sigma}_{1}$ & & & & & & $\mathrm{~T}$ (True) or F (False) \\
\hline & : & & & & & & $\mathrm{T}$ (True) or F (False) \\
\hline & $\tilde{\sigma}_{n}$ & & & & & & $\mathrm{~T}$ (True) or F (False) \\
\hline
\end{tabular}

Example 4. Let fuzzy DES be modeled by max-min automaton $\tilde{G}$ in Example 2, and $\operatorname{pr}(\tilde{K})$ is generated by $\tilde{H}$ in Example 3. By virtue of Fig. 6 we know that the all different pairs of fuzzy states reachable from $\left(\tilde{q}_{0}, \tilde{p}_{0}\right)$ are listed in TABLE III.

TABLE III. The computing tree visualized in Fig. 6 shows the all different pairs of fuzzy states reachable from $\left(\tilde{q}_{0}, \tilde{p}_{0}\right)$.

\begin{tabular}{|c|c|c|c|}
\hline$\tilde{s}$ & $\left(\tilde{q}_{0} \odot \tilde{s}, \tilde{p}_{0} \odot \tilde{s}\right)$ & $\tilde{s}$ & $\left(\tilde{q}_{0} \odot \tilde{s}, \tilde{p}_{0} \odot \tilde{s}\right)$ \\
\hline$\tilde{\epsilon}$ & $\left(\left[\begin{array}{ll}0.9 & 0.1\end{array}\right],\left[\begin{array}{ll}0.8 & 0.2\end{array}\right]\right)$ & $\tilde{\alpha}_{1} \tilde{\alpha}_{2}$ & $\left(\left[\begin{array}{ll}0.8 & 0.5\end{array}\right],\left[\begin{array}{ll}0.8 & 0.5\end{array}\right]\right)$ \\
\hline$\tilde{\alpha}_{1}$ & $\left(\left[\begin{array}{ll}0.4 & 0.8\end{array}\right],\left[\begin{array}{ll}0.2 & 0.8\end{array}\right]\right)$ & $\tilde{\alpha}_{1} \tilde{\alpha}_{2} \tilde{\alpha}_{2}$ & $\left(\left[\begin{array}{ll}0.5 & 0.5\end{array}\right],\left[\begin{array}{ll}0.5 & 0.5\end{array}\right]\right)$ \\
\hline$\tilde{\alpha}_{2}$ & $\left(\left[\begin{array}{ll}0.4 & 0.2\end{array}\right],\left[\begin{array}{ll}0.2 & 0.2\end{array}\right]\right)$ & $\tilde{\alpha}_{1} \tilde{\alpha}_{2} \tilde{\alpha}_{2} \tilde{\alpha}_{1}$ & $\left(\left[\begin{array}{ll}0.4 & 0.4\end{array}\right],\left[\begin{array}{ll}0.5 & 0.5\end{array}\right]\right)$ \\
\hline$\tilde{\alpha}_{1} \tilde{\alpha}_{1}$ & $\left(\left[\begin{array}{ll}0.4 & 0.4\end{array}\right],\left[\begin{array}{ll}0.2 & 0.2\end{array}\right]\right)$ & $\tilde{\alpha}_{1} \tilde{\alpha}_{2} \tilde{\alpha}_{2} \tilde{\alpha}_{1} \tilde{\alpha}_{2}$ & $\left(\left[\begin{array}{ll}0.4 & 0.4\end{array}\right],\left[\begin{array}{ll}0.5 & 0.5\end{array}\right]\right)$ \\
\hline
\end{tabular}

According to TABLE II, we can check the fuzzy controllability condition Eq. (9) of fuzzy DES in Example 4 via TABLE IV as follows (we only write the cases of $\tilde{s}=\epsilon$ and $\tilde{\alpha}_{1}$, since an "F" has been found up to now).

TABLE IV. Testing whether or not the fuzzy controllability condition Eq. (9) of fuzzy DES in Example 4 holds.
\begin{tabular}{|c|c|c|c|c|c|c|c|}
\hline$\tilde{s}$ & $\tilde{\sigma}$ & {$\left[\tilde{p}_{0} \odot \tilde{s}\right]$} & {$\left[\tilde{q}_{0} \odot \tilde{s} \odot \tilde{\sigma}\right]$} & $\tilde{\Sigma}_{u c}(\tilde{\sigma})$ & $L(\tilde{G}, \tilde{H}, \tilde{s}, \tilde{\sigma})$ & {$\left[\tilde{p}_{0} \odot \tilde{s} \odot \tilde{\sigma}\right]$} & $L(\tilde{G}, \tilde{H}, \tilde{s}, \tilde{\sigma}) \leq\left[\tilde{p}_{0} \odot \tilde{s} \odot \tilde{\sigma}\right]$ \\
\hline \multirow{2}{*}{$\tilde{\epsilon}$} & $\tilde{\alpha}_{1}$ & 0.8 & 0.8 & 0.7 & 0.7 & 0.8 & $\mathrm{~T}$ \\
\cline { 2 - 8 } & $\tilde{\alpha}_{2}$ & 0.8 & 0.4 & 0.2 & 0.2 & 0.2 & $\mathrm{~T}$ \\
\hline \multirow{2}{*}{$\tilde{\alpha}_{1}$} & $\tilde{\alpha}_{1}$ & 0.8 & 0.4 & 0.7 & 0.4 & 0.2 & $\mathrm{~F}$ \\
\cline { 2 - 8 } & $\tilde{\alpha}_{2}$ & 0.8 & 0.8 & 0.2 & 0.2 & 0.8 & $\mathrm{~T}$ \\
\hline
\end{tabular}

Therefore by means of TABLE IV we see that Eq. (9) does not hold for $\tilde{s}=\tilde{\alpha}_{1}$ and $\tilde{\sigma}=\tilde{\alpha}_{1}$.

\section{A Primary Application to Medicine: A Formal Example}

We further state some applicable background of fuzzy DESs. Fuzzy control and fuzzy systems in biomedical engineering have been significantly studied (for example, see [6]), in which fuzzy 
drug delivery system for real-time control of mean arterial pressure (MAP), cardiac output $(\mathrm{CO})$, and mean pulmonary arterial pressure (MPAP) in patients is one of the main concerns. The heart patient's status may be represented via the degrees of the three hemodynamic variables, i.e., MAP, CO, and MPAP, and they may be low, high, or normal. These drugs such as dopamine (DPM), sodium nitroprusside (SNP), nitroglycerin (NTG), and phenylephrine (PNP) are appropriately used to regulate the degrees of MAP, CO, and MPAP. It may be logically modeled via fuzzy DESs with supervisory control, in which the uses of the dosages of DPM, SNP, NTG, and PNP may be thought of as some fuzzy events. But here we consider the patient's condition roughly to be three cases, i.e., "poor", "fair", and "excellent", and, as a first step, apply the above results regarding supervised control of fuzzy DESs to control the three states. To this end, we further describe the following examples, which may be viewed as an applicable background of supervisory control of fuzzy DESs.
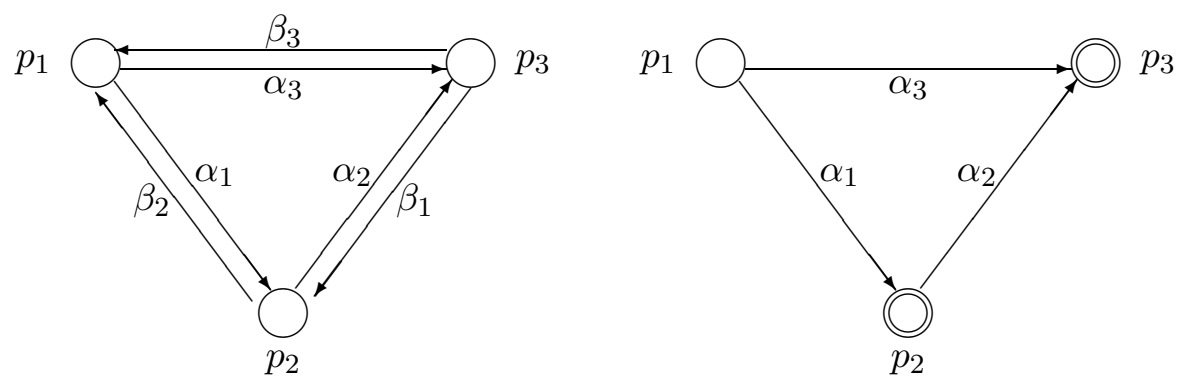

Fig. 7. Finite automaton modelling patient's heart condition. Fig. 8. Finite automaton modelling the given specifications.

Example 5. Let us use automaton $G=\left(Q, \Sigma, \delta, p_{1}\right)$ to model a patient's heart condition, where $Q=\left\{p_{1}, p_{2}, p_{3}\right\}, \Sigma=\left\{\alpha_{1}, \alpha_{2}, \alpha_{3}, \beta_{1}, \beta_{2}, \beta_{3}\right\}$, and $\delta$ is visualized by Fig. 7. Here $p_{1}=P, p_{2}=F$, and $p_{3}=E$ represent "poor", "fair", and "excellent", respectively. A patient's initial condition may be "poor" and should become "fair" and even "excellent" after certain treatment. When patient's health gets "fair", we naturally hope it to be better and better, say "excellent", instead of deteriorating, i.e., recurring to "poor". Analogously, if the patient's condition has been "excellent", it is desired to keep the good health and thus a supervisor is necessary to disable the events $\beta_{1}, \beta_{2}$, and $\beta_{3}$ in case they are controllable. Let us assume $\Sigma_{u c}=\left\{\alpha_{1}, \alpha_{2}, \alpha_{3}\right\}, \Sigma_{c}=\left\{\beta_{1}, \beta_{2}, \beta_{3}\right\}$, and the given set of specifications $K=\left\{\alpha_{1}, \alpha_{1} \alpha_{2}, \alpha_{3}\right\}$ as desired. Actually, $K$ is marked by automaton $H=\left(Q, \Sigma_{u c}, \delta_{1}, p_{1}, Q_{m}\right)$ depicted by Fig. 8, where $Q_{m}=\left\{p_{2}, p_{3}\right\}$. Clearly, $\operatorname{pr}(K)=\left\{\epsilon, \alpha_{1}, \alpha_{1} \alpha_{2}, \alpha_{3}\right\}$ is generated by $H$. In terms of the method described above for checking the controllability condition Eq. (9), it is readily seen that $K$ is controllable with respect to $L(G)$ and $\Sigma_{u c}$, that is, $\operatorname{pr}(K) \Sigma_{u c} \cap L(G) \subseteq \operatorname{pr}(K)$ holds, so by virtue of crisp controllable theorem indicated above, there is a supervisor $S: \Sigma^{*} \rightarrow \mathcal{P}(\Sigma)$ such that $L(S / G)=\operatorname{pr}(K)$, where $S$ is defined as: For 
any $s \in \operatorname{pr}(K), S(s)=\Gamma_{H}\left(\delta_{1}\left(p_{1}, s\right)\right)$. Here symbol $\Gamma_{H}(q)$ denotes the set of active events in the current state $q$. More explicitly, $S(\epsilon)=\left\{\alpha_{1}, \alpha_{3}\right\}, S\left(\alpha_{1}\right)=\left\{\alpha_{2}\right\}, S\left(\alpha_{1} \alpha_{2}\right)=\emptyset, S\left(\alpha_{3}\right)=\emptyset$.

In real-life situation, a patient's condition can simultaneously belong to "excellent", "fair", and even "poor" with respective memberships; also, an event occurring (i.e., a treatment) may lead a state to multi-states with respective degrees. Therefore, a patient's conditions and their changes after certain treatments should be modeled by a max-min automaton $\tilde{G}=\left(\tilde{Q}, \tilde{\Sigma}, \tilde{\delta}, \tilde{q}_{0}\right)$, and the fuzzy events $\tilde{\alpha}_{i}, \tilde{\beta}_{i}(i=1,2)$ may be evaluated as follows by means of some diagnosis together with medical theory and experience (for example, by virtue of an intelligent system designed for automating drug delivery in [6, page 323], which is described by Fig. 9, and to a certain extent, similar to the process of supervisory control of fuzzy DESs).

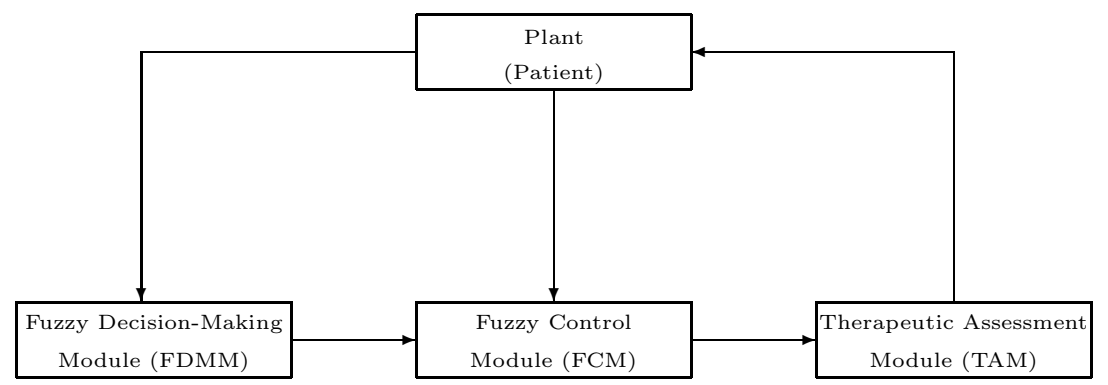

$$
\begin{aligned}
& \tilde{\alpha}_{1}=\left[\begin{array}{ccc}
0.4 & 0.9 & 0.4 \\
0 & 0.4 & 0.4 \\
0 & 0 & 0.4
\end{array}\right], \quad \tilde{\alpha}_{2}=\left[\begin{array}{ccc}
0.4 & 0.4 & 0.4 \\
0 & 0.4 & 0.9 \\
0 & 0 & 0.4
\end{array}\right], \quad \tilde{\alpha}_{3}=\left[\begin{array}{ccc}
0.4 & 0.4 & 0.9 \\
0 & 0.4 & 0.4 \\
0 & 0 & 0.4
\end{array}\right] \text {, } \\
& \tilde{\beta}_{1}=\left[\begin{array}{ccc}
0.4 & 0 & 0 \\
0.4 & 0.4 & 0 \\
0.4 & 0.9 & 0.4
\end{array}\right], \quad \tilde{\beta}_{2}=\left[\begin{array}{ccc}
0.4 & 0 & 0 \\
0.9 & 0.4 & 0 \\
0.4 & 0.4 & 0.4
\end{array}\right], \quad \tilde{\beta}_{3}=\left[\begin{array}{ccc}
0.4 & 0 & 0 \\
0.4 & 0.4 & 0 \\
0.9 & 0.4 & 0.4
\end{array}\right] ;
\end{aligned}
$$

$\tilde{\delta}$ is defined as before; $\tilde{q}_{0}=\left[\begin{array}{lll}0.9 & 0.1 & 0\end{array}\right]$. As analyzed above, we hope the patient's condition to be better and better. To this end, we specify a fuzzy set of control specifications $\tilde{K} \in \mathcal{F}\left(\tilde{\Sigma}^{*}\right)$ that are desired, and show whether there exists a supervisor that can disable some fuzzy events with respective degrees such that the restricted behavior of the supervised fuzzy system satisfies those given specifications.

As usual, let $\operatorname{pr}(\tilde{K})$ be generated by a max-min automaton $\tilde{H}=\left(\tilde{Q}, \tilde{\Sigma}, \tilde{\delta}_{1}, \tilde{q}_{0}\right)$, that is assumed to be derived from an intelligent system mentioned above, where $\tilde{q}_{0}=\left[\begin{array}{lll}0.9 & 0.1 & 0\end{array}\right]$; 
$\tilde{\Sigma}=\left\{\tilde{\alpha}_{1}, \tilde{\alpha}_{2}, \tilde{\alpha}_{3}, \tilde{\beta}_{1}, \tilde{\beta}_{2}, \tilde{\beta}_{3}\right\}$ where $\tilde{\alpha}_{1}, \tilde{\alpha}_{2}, \tilde{\alpha}_{3}$ are the same as those in $\tilde{G}$, but $\tilde{\beta}_{1}, \tilde{\beta}_{2}, \tilde{\beta}_{3}$ are changed as follows:

$$
\tilde{\beta}_{1}=\left[\begin{array}{ccc}
0.2 & 0 & 0 \\
0.2 & 0.2 & 0 \\
0.2 & 0.9 & 0.2
\end{array}\right], \quad \tilde{\beta}_{2}=\left[\begin{array}{ccc}
0.2 & 0 & 0 \\
0.9 & 0.2 & 0 \\
0.2 & 0.2 & 0.2
\end{array}\right], \quad \tilde{\beta}_{3}=\left[\begin{array}{ccc}
0.2 & 0 & 0 \\
0.2 & 0.2 & 0 \\
0.9 & 0.2 & 0.2
\end{array}\right] ;
$$

$\tilde{\delta}_{1}$ is naturally defined in terms of these given fuzzy events. Clearly, $\operatorname{pr}(\tilde{K})=\mathcal{L}_{\tilde{H}} \subseteq \mathcal{L}_{\tilde{G}}$ since each element in every fuzzy event of $\tilde{H}$ is not bigger than the corresponding element in the same fuzzy event of $\tilde{G}$. By means of the computing tree described by Fig. 6, we can obtain that there are only twelve different pairs of fuzzy states reachable from $\left(\tilde{q}_{0}, \tilde{q}_{0}\right)$, that are listed in TABLE V.

TABLE V. The all different pairs of fuzzy states reachable from $\left(\tilde{q}_{0}, \tilde{q}_{0}\right)$.

\begin{tabular}{|c|c|c|c|c|c|c|c|}
\hline$\tilde{s}$ & $\left(\tilde{q}_{0} \odot \tilde{s}, \quad \tilde{q}_{0} \odot \tilde{s}\right)$ & $\tilde{s}$ & \multicolumn{5}{|c|}{$\left(\tilde{q}_{0} \odot \tilde{s}, \quad \tilde{q}_{0} \odot \tilde{s}\right)$} \\
\hline$\tilde{\epsilon}$ & $\left(\left[\begin{array}{lll}0.9 & 0.1 & 0\end{array}\right],\left[\begin{array}{lll}0.9 & 0.1 & 0\end{array}\right]\right)$ & $\begin{array}{c}\tilde{\alpha}_{1} \tilde{\beta}_{2} \\
\end{array}$ & $([0.9$ & 0.4 & $0.4],[0.9$ & 0.2 & $0.2])$ \\
\hline$\tilde{\alpha}_{1}$ & $\left(\left[\begin{array}{lll}0.4 & 0.9 & 0.4\end{array}\right],\left[\begin{array}{lll}0.4 & 0.9 & 0.4\end{array}\right]\right)$ & $\tilde{\alpha}_{2} \tilde{\beta}_{2}$ & $([0.4$ & 0.4 & $0.4],[0.4$ & 0.2 & $0.2])$ \\
\hline$\tilde{\alpha}_{2}$ & $\left(\left[\begin{array}{lll}0.4 & 0.4 & 0.4\end{array}\right],\left[\begin{array}{lll}0.4 & 0.4 & 0.4\end{array}\right]\right)$ & $\tilde{\alpha}_{3} \tilde{\beta}_{1}$ & $([0.4$ & 0.9 & $0.4],[0.2$ & 0.9 & 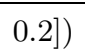 \\
\hline$\tilde{\alpha}_{3}$ & $\left(\left[\begin{array}{lll}0.4 & 0.4 & 0.9\end{array}\right],\left[\begin{array}{lll}0.4 & 0.4 & 0.9\end{array}\right]\right)$ & $\overline{\alpha_{3}} \tilde{\beta}_{1} \tilde{\alpha}_{1}$ & $\overline{([0.4}$ & 0.4 & $0.4],[0.2$ & 0.4 & $0.4])$ \\
\hline$\tilde{\beta}_{1}$ & $\left(\left[\begin{array}{lll}0.4 & 0.1 & 0\end{array}\right],\left[\begin{array}{lll}0.2 & 0.1 & 0\end{array}\right]\right)$ & $\tilde{\alpha}_{3} \tilde{\beta}_{1} \tilde{\alpha}_{2}$ & $\overline{([0.4}$ & 0.4 & $0.9],[0.2$ & 0.4 & 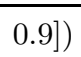 \\
\hline$\tilde{\alpha}_{1} \tilde{\beta}_{1}$ & $\left(\left[\begin{array}{lll}0.4 & 0.4 & 0.4\end{array}\right],\left[\begin{array}{lll}0.2 & 0.2 & 0.2\end{array}\right]\right)$ & $\tilde{\alpha}_{3} \tilde{\beta}_{1} \tilde{\alpha}_{1} \tilde{\beta}_{1}$ & $([0.4$ & 0.4 & $0.4],[0.2$ & 0.4 & $0.2])$ \\
\hline
\end{tabular}

Suppose that the fuzzy set of uncontrollable events $\tilde{\Sigma}_{u c}$ is also evaluated in terms of medical theory and experience as: $\tilde{\Sigma}_{u c}\left(\tilde{\alpha_{1}}\right)=0.8 ; \tilde{\Sigma}_{u c}\left(\tilde{\alpha_{2}}\right)=0.75 ; \tilde{\Sigma}_{u c}\left(\tilde{\alpha_{3}}\right)=0.7 ; \tilde{\Sigma}_{u c}\left(\tilde{\beta}_{1}\right)=$ $0.2 ; \tilde{\Sigma}_{u c}\left(\tilde{\beta}_{2}\right)=0.25 ; \tilde{\Sigma}_{u c}\left(\tilde{\beta}_{3}\right)=0.3$; and $\tilde{\Sigma}_{c}$ is naturally decided due to $\tilde{\Sigma}_{u c}(\tilde{\sigma})+\tilde{\Sigma}_{c}(\tilde{\sigma})=1$ for any fuzzy event $\tilde{\sigma} \in\left\{\tilde{\alpha_{1}}, \tilde{\alpha_{2}}, \tilde{\alpha_{3}}, \tilde{\beta}_{1}, \tilde{\beta_{2}}, \tilde{\beta_{3}}\right\}$. Then by virtue of TABLE I we can test that the fuzzy controllability condition Eq. (9) does not hold for $\tilde{s}=\epsilon, \tilde{\sigma}=\tilde{\beta}_{2}$ or $\tilde{\beta}_{3}$. If $\tilde{\Sigma}_{u c} \leq 0.2$ for any $\tilde{\sigma} \in \tilde{\Sigma}$, then it can be checked by using TABLE I that Eq. (9) holds true, and in terms of Theorem 1 we know that the supervisor $\tilde{S}$ yielding $\operatorname{pr}(\tilde{K})$ therefore exists. In practice, the degree of each fuzzy event being controlled by the supervisor is specified according to Eq. (10), and the therapeutic process is then implemented under the control of the supervisor, which results in the desired specification $\operatorname{pr}(\tilde{K})$.

\section{Nonblocking Controllability Theorem for Fuzzy DESs}

In supervisory control of crisp DESs, nonblockingness is usually required and it means that the production sequence is completed without deadlock, which has been applied to manufacturing to avoid deadlock. In reality, analogous restriction is sometimes imposed on fuzzy DESs. More 
exactly, the specifications on that controlled system are given as a sublanguage of $\mathcal{L}_{\tilde{G}, m}$, and the supervisor $\tilde{S}$ is nonblocking, that is,

$$
\operatorname{pr}\left(\mathcal{L}_{\tilde{S} / \tilde{G}, m}\right)=\mathcal{L}_{\tilde{S} / \tilde{G}}
$$

otherwise, $\tilde{S}$ is blocking. Intuitively, $\tilde{S}$ being nonblocking means that for any string of fuzzy events $\tilde{s}$, the degree to which $\tilde{s}$ belongs to the fuzzy language generated by the supervised fuzzy system $\tilde{S} / \tilde{G}$ equals the membership degree of $\tilde{s}$ belonging to the prefix-closure of the fuzzy language marked by the supervised fuzzy system $\tilde{S} / \tilde{G}$. In Example 5 , if the state "excellent" is indicated as a marked state, then the nonblockingness renders that any partial therapeutic process (say $\tilde{\alpha}_{1}$ ) being implemented under the supervisor $\tilde{S}$ has the possibility not more than that of the whole expected therapeutic strategy (say $\tilde{\alpha}_{1} \tilde{\alpha}_{2}$ ) supervised by $\tilde{S}$ being realized, which also conforms to the crisp situation. We provide another numeric example to serve as further illustration.

Example 6. Let set of fuzzy events $\tilde{\Sigma}=\{\tilde{a}, \tilde{b}, \tilde{c}\}$. Assume that the fuzzy language $\mathcal{L}_{\tilde{G}}$ and $\mathcal{L}_{\tilde{G}, m}$ generated and marked by a fuzzy finite automaton (max-min or max-product) $\tilde{G}$ are respectively as follows: $\mathcal{L}_{\tilde{G}}(\epsilon)=1, \mathcal{L}_{\tilde{G}}(\tilde{a})=\mathcal{L}_{\tilde{G}}(\tilde{a} \tilde{b})=\mathcal{L}_{\tilde{G}}(\tilde{a} \tilde{b} \tilde{c})=0.8$, and $\mathcal{L}_{\tilde{G}}(\tilde{s})=0$ for the other $s \in \tilde{\Sigma}^{*} ; \mathcal{L}_{\tilde{G}, m}(\epsilon)=1, \mathcal{L}_{\tilde{G}, m}(\tilde{a} \tilde{b})=0.8$, and $\mathcal{L}_{\tilde{G}, m}(\tilde{s})=0$ for the other $s \in \tilde{\Sigma}^{*}$. If $\tilde{\Sigma}_{u c}(\tilde{a})=\tilde{\Sigma}_{u c}(\tilde{b})=0.8$ and $\tilde{\Sigma}_{u c}(\tilde{c})=0$, we define a supervisor $\tilde{S}: \tilde{\Sigma}^{*} \rightarrow \mathcal{F}(\tilde{\Sigma})$ as follows: $\tilde{S}(\epsilon)(\tilde{a})=1, \tilde{S}(\tilde{a})(\tilde{b})=0.8, \tilde{S}(\tilde{a} \tilde{b})(\tilde{c})=0$, and also 0 for the other cases. Then it is easy to check that $\tilde{S}$ satisfies the fuzzy admissibility condition described by Eq. (6), and

$$
\begin{gathered}
\mathcal{L}_{\tilde{S} / \tilde{G}}(\tilde{a})=\operatorname{pr}\left(\mathcal{L}_{\tilde{S} / \tilde{G}, m}\right)(\tilde{a})=0.8, \\
\mathcal{L}_{\tilde{S} / \tilde{G}}(\tilde{a} \tilde{b})=\operatorname{pr}\left(\mathcal{L}_{\tilde{S} / \tilde{G}, m}\right)(\tilde{a} \tilde{b})=0.8,
\end{gathered}
$$

and $\mathcal{L}_{\tilde{S} / \tilde{G}}(\tilde{s})=\operatorname{pr}\left(\mathcal{L}_{\tilde{S} / \tilde{G}, m}\right)(\tilde{s})=0$ for the other $\tilde{s} \in \tilde{\Sigma}^{*}$. Therefore, in this case, $\tilde{S}$ is nonblocking. However, if $\tilde{\Sigma}_{u c}(\tilde{c})>0$, and still $\tilde{\Sigma}_{u c}(\tilde{a})=\tilde{\Sigma}_{u c}(\tilde{b})=0.8$, then from the fuzzy admissibility condition it follows that

$$
\tilde{S}(\tilde{a} \tilde{b})(\tilde{c}) \geq \min \left\{\tilde{\Sigma}_{u c}(\tilde{c}), \mathcal{L}_{\tilde{G}}(\tilde{a} \tilde{b} \tilde{c})\right\}=\min \left\{\tilde{\Sigma}_{u c}(\tilde{c}), 0.8\right\}>0
$$

and thus $\mathcal{L}_{\tilde{S} / \tilde{G}}(\tilde{a} \tilde{b} \tilde{c})=\min \left\{\Sigma_{u c}(\tilde{c}), 0.8\right\}$, but $\operatorname{pr}\left(\mathcal{L}_{\tilde{S} / \tilde{G}, m}\right)(\tilde{a} \tilde{b} \tilde{c})=0$. Therefore in this case $\operatorname{pr}\left(\mathcal{L}_{\tilde{S} / \tilde{G}, m}\right) \neq \mathcal{L}_{\tilde{S} / \tilde{G}}$, which shows that the supervisor $\tilde{S}$ is blocking.

In this regard, we have the nonblocking controllability theorem of fuzzy DESs.

Theorem 2. Let a fuzzy DES be modeled by fuzzy finite automaton (max-min or maxproduct) $\tilde{G}=\left(\tilde{Q}, \tilde{\Sigma}, \tilde{\delta}, \tilde{q}_{0}, \tilde{Q}_{m}\right)$, and let $\tilde{\Sigma}_{u c} \in \mathcal{F}(\tilde{\Sigma})$ be the fuzzy uncontrollable subset of $\tilde{\Sigma}$. 
Suppose fuzzy language $\tilde{K} \subseteq \mathcal{L}_{\tilde{G}, m}$ satisfying $\tilde{K}(\epsilon)=1$ and $\operatorname{pr}(\tilde{K}) \subseteq \mathcal{L}_{\tilde{G}, m}$. Then there exists a nonblocking supervisor $\tilde{S}$ for $\tilde{G}$ such that $\tilde{S}$ satisfies the fuzzy admissibility condition Eq. (6), and

$$
\mathcal{L}_{\tilde{S} / \tilde{G}, m}=\tilde{K} \text { and } \mathcal{L}_{\tilde{S} / \tilde{G}}=\operatorname{pr}(\tilde{K})
$$

if and only if $\tilde{K}=\operatorname{pr}(\tilde{K}) \tilde{\cap} \mathcal{L}_{\tilde{G}, m}$ and the fuzzy controllability condition of $\tilde{K}$ with respect to $\tilde{G}$ and $\tilde{\Sigma}_{u c}$ holds, i.e., Eq. (9) holds.

Proof: The proof of sufficiency is similar to that of Theorem 1. Set $\tilde{S}: \tilde{\Sigma}^{*} \rightarrow \mathcal{F}(\tilde{\Sigma})$ as Eq. (10): For any $\tilde{s} \in \tilde{\Sigma}^{*}$ and any $\tilde{\sigma} \in \tilde{\Sigma}$,

$$
\tilde{S}(\tilde{s})(\tilde{\sigma})= \begin{cases}\min \left\{\tilde{\Sigma}_{u c}(\tilde{\sigma}), \mathcal{L}_{\tilde{G}}(\tilde{s} \tilde{\sigma})\right\}, & \text { if } \tilde{\Sigma}_{u c}(\tilde{\sigma}) \geq \operatorname{pr}(\tilde{K})(\tilde{s} \tilde{\sigma}) \\ \operatorname{pr}(\tilde{K})(\tilde{s} \tilde{\sigma}), & \text { otherwise. }\end{cases}
$$

Then with the same process as Theorem 1 we can prove that $\mathcal{L}_{\tilde{S} / \tilde{G}}=\operatorname{pr}(\tilde{K})$. Furthermore, since $\tilde{K}=\operatorname{pr}(\tilde{K}) \tilde{\cap} \mathcal{L}_{\tilde{G}, m}$, we also have that

$$
\mathcal{L}_{\tilde{S} / \tilde{G}, m}=\mathcal{L}_{\tilde{S} / \tilde{G}} \tilde{\cap} \mathcal{L}_{\tilde{G}, m}=\operatorname{pr}(\tilde{K}) \tilde{\cap} \mathcal{L}_{\tilde{G}, m}=\tilde{K}
$$

by which and $\mathcal{L}_{\tilde{S} / \tilde{G}}=\operatorname{pr}(\tilde{K})$ we obtain $\operatorname{pr}\left(\mathcal{L}_{\tilde{S} / \tilde{G}, m}\right)=\mathcal{L}_{\tilde{S} / \tilde{G}}$, i.e., $\tilde{S}$ is nonblocking.

On the other hand, given that there exists an nonblocking supervisory $\tilde{S}$ such that

$$
\mathcal{L}_{\tilde{S} / \tilde{G}, m}=\tilde{K} \text { and } \mathcal{L}_{\tilde{S} / \tilde{G}}=\operatorname{pr}(\tilde{K})
$$

then with the definition of $\mathcal{L}_{\tilde{S} / \tilde{G}, m}$ we have that

$$
\tilde{K}=\mathcal{L}_{\tilde{S} / \tilde{G}, m}=\mathcal{L}_{\tilde{S} / \tilde{G}} \tilde{\cap} \mathcal{L}_{\tilde{G}, m}=\operatorname{pr}(\tilde{K}) \tilde{\cap} \mathcal{L}_{\tilde{G}, m} .
$$

As well, the proof of fuzzy controllability condition is really analogous to that of Theorem 1. Therefore, we complete the proof of this theorem.

Remark 6. In Theorem 2, condition $\operatorname{pr}(\tilde{K}) \subseteq \mathcal{L}_{\tilde{G}, m}$ is necessary since $\tilde{K} \subseteq \mathcal{L}_{\tilde{G}, m}$ does not always result in $\operatorname{pr}(\tilde{K}) \subseteq \mathcal{L}_{\tilde{G}, m}$. (In Theorem $1 \operatorname{pr}(\tilde{K}) \subseteq \mathcal{L}_{\tilde{G}}$ is not prerequisite, because it was shown that $\tilde{K} \subseteq \mathcal{L}_{\tilde{G}}$ deduces $\operatorname{pr}(\tilde{K}) \subseteq \mathcal{L}_{\tilde{G}}$.

Example 7. Let a fuzzy DES be modeled by a fuzzy finite automaton $\tilde{G}=\left(\tilde{Q}, \tilde{\Sigma}, \tilde{\delta}, \tilde{q}_{0}, \tilde{Q}_{m}\right)$ where $\tilde{\Sigma}=\{\tilde{a}, \tilde{b}, \tilde{c}\}$. The fuzzy languages $\mathcal{L}_{\tilde{G}}$ and $\mathcal{L}_{\tilde{G}, m}$ over $\tilde{\Sigma}$ generated and marked by the fuzzy finite automaton $\tilde{G}$ are the same as those in Example 6, that is, $\mathcal{L}_{\tilde{G}}(\epsilon)=1$, $\mathcal{L}_{\tilde{G}}(\tilde{a})=\mathcal{L}_{\tilde{G}}(\tilde{a} \tilde{b})=\mathcal{L}_{\tilde{G}}(\tilde{a} \tilde{b} \tilde{c})=0.8$, and $\mathcal{L}_{\tilde{G}}(\tilde{s})=0$ for the other $s \in \tilde{\Sigma}^{*} ; \mathcal{L}_{\tilde{G}, m}(\epsilon)=1$, $\mathcal{L}_{\tilde{G}, m}(\tilde{a} \tilde{b})=0.8$, and $\mathcal{L}_{\tilde{G}, m}(\tilde{s})=0$ for the other $s \in \tilde{\Sigma}^{*}$. Assume that a fuzzy set of control 
specifications $\tilde{K}$ is defined as: $\tilde{K}(\epsilon)=1, \tilde{K}(\tilde{a} \tilde{b})=0.8$, and $\tilde{K}(\tilde{s})=0$ for the other $\tilde{s} \in \tilde{\Sigma}^{*}$. Clearly, $\operatorname{pr}(\tilde{K}) \subseteq \mathcal{L}_{\tilde{G}, m}$ and $\tilde{K}=\operatorname{pr}(\tilde{K}) \tilde{\cap} \mathcal{L}_{\tilde{G}, m}$. Also, it is readily checked that the fuzzy controllability condition of $\tilde{K}$ with respect to $\tilde{G}$ and $\tilde{\Sigma}_{u c}$ holds. Therefore, by means of Theorem 2 there is a supervisor $\tilde{S}$ such that the supervised fuzzy control system $\mathcal{L}_{\tilde{S} / \tilde{G}}$ satisfies

$$
\mathcal{L}_{\tilde{S} / \tilde{G}, m}=\tilde{K} \text { and } \mathcal{L}_{\tilde{S} / \tilde{G}}=\operatorname{pr}(\tilde{K}) .
$$

From the proof of Theorem 2 we know that supervisor $\tilde{S}: \tilde{\Sigma}^{*} \rightarrow \mathcal{F}(\tilde{\Sigma})$ is defined as Eq. (10). More concretely, in terms of Eq. (10) we have that $\tilde{S}(\epsilon)(\tilde{a})=\tilde{S}(\tilde{a})(\tilde{b})=0.8$, and 0 for the other cases. It is seen that the deduced $\tilde{S}$ coincides exactly with that in Example 6 .

Similarly to Corollary 1, with Theorem 2 we have:

Corollary 2. Let a fuzzy DES be modeled by fuzzy finite automaton (max-min or maxproduct) $\tilde{G}=\left(\tilde{Q}, \tilde{\Sigma}, \tilde{\delta}, \tilde{q}_{0}, \tilde{Q}_{m}\right)$. Let $n$ be any given positive integer. Suppose fuzzy uncontrollable subset $\tilde{\Sigma}_{u c} \in \mathcal{F}(\tilde{\Sigma})$, and fuzzy legal subset $\tilde{K} \in \mathcal{F}\left(\tilde{\Sigma}^{*}\right)$ that satisfies: $\tilde{K} \subseteq_{n} \mathcal{L}_{\tilde{G}}$, $\operatorname{pr}(\tilde{K}) \subseteq_{n} \mathcal{L}_{\tilde{G}, m}$, and $\tilde{K}(\epsilon)=1$. Then there exists a nonblocking supervisor $\tilde{S}$ for $\tilde{G}$ such that $\tilde{S}$ satisfies the fuzzy $n$-admissibility condition Eq. (6) and

$$
\mathcal{L}_{\tilde{S} / \tilde{G}, m}={ }_{n} \tilde{K} \text { and } \mathcal{L}_{\tilde{S} / \tilde{G}}={ }_{n} \operatorname{pr}(\tilde{K})
$$

if and only if $\tilde{K}={ }_{n} \operatorname{pr}(\tilde{K}) \tilde{\cap} \mathcal{L}_{\tilde{G}, m}$ and the fuzzy $n$-controllability condition of $\tilde{K}$ with respect to $\tilde{G}$ and $\tilde{\Sigma}_{u c}$ holds, i.e., Eq. (13) holds.

Proof: It is the same process as Theorem 2 by restricting the length of $\tilde{s}$ with $|\tilde{s}| \leq n$.

\section{Properties of Controllability of Fuzzy DESs}

In this section, we deal with a number of basic properties concerning supervisory controllability in fuzzy DESs.

Definition 1. Let $\tilde{K}$ and $\tilde{M}$ be fuzzy languages over set $\tilde{\Sigma}$ of fuzzy events, and $\operatorname{pr}(\tilde{M})=\tilde{M}$. Suppose that $\tilde{\Sigma}_{u c} \in \mathcal{F}(\tilde{\Sigma})$ denotes a fuzzy subset of uncontrollable events. Then $\tilde{K}$ is said to be controllable with respect to $\tilde{M}$ and $\tilde{\Sigma}_{u c}$ if for any $\tilde{s} \in \tilde{\Sigma}^{*}$ and any $\tilde{\sigma} \in \tilde{\Sigma}$,

$$
\min \left\{\operatorname{pr}(\tilde{K})(\tilde{s}), \tilde{\Sigma}_{u c}(\tilde{\sigma}), \tilde{M}(\tilde{s} \tilde{\sigma})\right\} \leq \operatorname{pr}(\tilde{K})(\tilde{s} \tilde{\sigma}) .
$$

Intuitively, Eq. (19) means that the degree to which string $\tilde{s}$ belongs to the prefix-closure of fuzzy language $\tilde{K}$ and fuzzy event string $\tilde{\sigma} \tilde{s}$ also belongs to fuzzy language $\tilde{M}$ together 
with $\tilde{\sigma}$ being uncontrollable is not bigger than the possibility of the string $\tilde{s} \tilde{\sigma}$ pertaining to the prefix-closure of $\tilde{K}$. For convenience, we denote by $C\left(\tilde{M}, \tilde{\Sigma}_{u c}\right)$ the set of all those being controllable respect to $\tilde{M}$ and $\tilde{\Sigma}_{u c}$, that is,

$$
C\left(\tilde{M}, \tilde{\Sigma}_{u c}\right)=\left\{\tilde{L} \in \mathcal{F}\left(\tilde{\Sigma}^{*}\right): \tilde{L} \text { is controllable with respect to } \tilde{M} \text { and } \tilde{\Sigma}_{u c}\right\}
$$

Clearly, when $\tilde{M}$ is generated by some fuzzy finite automaton $\tilde{G}$, i.e., $\tilde{M}=\mathcal{L}_{\tilde{G}}$, then Eq. (19) is the same as the fuzzy controllable condition described by Eq. (9) in Theorem 1. Also, it is easy to see that $\tilde{K} \in C\left(\tilde{M}, \tilde{\Sigma}_{u c}\right)$ implies $\operatorname{pr}(\tilde{K}) \in C\left(\tilde{M}, \tilde{\Sigma}_{u c}\right)$.

Proposition 1. Let $\tilde{M} \in \mathcal{F}\left(\tilde{\Sigma}^{*}\right)$ and $\tilde{\Sigma}_{u c} \in \mathcal{F}(\tilde{\Sigma})$ be the same as those in Definition 1. Suppose that $\tilde{K}_{1}, \tilde{K}_{2}$ are fuzzy languages over set $\tilde{\Sigma}$ of fuzzy events. Then:

(i) If $\tilde{K}_{1}, \tilde{K}_{2} \in C\left(\tilde{M}, \tilde{\Sigma}_{u c}\right)$, then so is $\tilde{K}_{1} \tilde{\cup} \tilde{K}_{2}$, where $\tilde{\cup}$ is Zadeh fuzzy OR operator, that is, $(\tilde{A} \tilde{\cup} \tilde{B})(x)=\max \{\tilde{A}(x), \tilde{B}(x)\}$.

(ii) If $\operatorname{pr}\left(\tilde{K}_{1}\right) \tilde{\cap} \operatorname{pr}\left(\tilde{K}_{2}\right)=\operatorname{pr}\left(\tilde{K}_{1} \tilde{\cap}_{2}\right)$, and $\tilde{K}_{1}$ and $\tilde{K}_{2} \in C\left(\tilde{M}, \tilde{\Sigma}_{u c}\right)$, then $\tilde{K}_{1} \tilde{\cap} \tilde{K}_{2} \in$ $C\left(\tilde{M}, \tilde{\Sigma}_{u c}\right)$, too.

(iii) If $\operatorname{pr}\left(\tilde{K}_{i}\right)=\tilde{K}_{i}, i=1,2$, and both $\tilde{K}_{1}$ and $\tilde{K}_{2} \in C\left(\tilde{M}, \tilde{\Sigma}_{u c}\right)$, then $\operatorname{pr}\left(\tilde{K}_{1} \tilde{\cap} \tilde{K}_{2}\right)=$ $\tilde{K}_{1} \tilde{\cap}_{2}$ and $\tilde{K}_{1} \tilde{\cap} \tilde{K}_{2} \in C\left(\tilde{M}, \tilde{\Sigma}_{u c}\right)$.

Proof: (i) For any $\tilde{s} \in \tilde{\Sigma}^{*}$ and any $\tilde{\sigma} \in \tilde{\Sigma}$, we have

$$
\begin{aligned}
& \min \left\{\operatorname{pr}\left(\tilde{K}_{1} \tilde{\cup} \tilde{K}_{2}\right)(\tilde{s}), \tilde{\Sigma}_{u c}(\tilde{\sigma}), \tilde{M}(\tilde{s} \tilde{\sigma})\right\} \\
= & \min \left\{\max \left\{\operatorname{pr}\left(\tilde{K}_{1}\right)(\tilde{s}), \operatorname{pr}\left(\tilde{K}_{2}\right)(\tilde{s})\right\}, \tilde{\Sigma}_{u c}(\tilde{\sigma}), \tilde{M}(\tilde{s} \tilde{\sigma})\right\} \\
= & \max \left\{\min \left\{\operatorname{pr}\left(\tilde{K}_{1}\right)(\tilde{s}), \tilde{\Sigma}_{u c}(\tilde{\sigma}), \tilde{M}(\tilde{s} \tilde{\sigma})\right\}, \min \left\{\operatorname{pr}\left(\tilde{K}_{1}\right)(\tilde{s}), \tilde{\Sigma}_{u c}(\tilde{\sigma}), \tilde{M}(\tilde{s} \tilde{\sigma})\right\}\right\} \\
\leq & \max \left\{\operatorname{pr}\left(\tilde{K}_{1}\right)(\tilde{s} \tilde{\sigma}), \operatorname{pr}\left(\tilde{K}_{2}\right)(\tilde{s} \tilde{\sigma})\right\} \\
= & \operatorname{pr}\left(\tilde{K}_{1} \tilde{\cup} \tilde{K}_{2}\right)(\tilde{s} \tilde{\sigma}) .
\end{aligned}
$$

(ii) For any $\tilde{s} \in \tilde{\Sigma}^{*}$ and any $\tilde{\sigma} \in \tilde{\Sigma}$, we obtain that

$$
\begin{aligned}
& \min \left\{\operatorname{pr}\left(\tilde{K}_{1} \tilde{\cap} \tilde{K}_{2}\right)(\tilde{s}), \tilde{\Sigma}_{u c}(\tilde{\sigma}), \tilde{M}(\tilde{s} \tilde{\sigma})\right\} \\
\leq & \min \left\{\min \left\{\operatorname{pr}\left(\tilde{K}_{1}\right)(\tilde{s}), \operatorname{pr}\left(\tilde{K}_{2}\right)(\tilde{s})\right\}, \tilde{\Sigma}_{u c}(\tilde{\sigma}), \tilde{M}(\tilde{s} \tilde{\sigma})\right\} \\
= & \min \left\{\min \left\{\operatorname{pr}\left(\tilde{K}_{1}\right)(\tilde{s}), \tilde{\Sigma}_{u c}(\tilde{\sigma}), \tilde{M}(\tilde{s} \tilde{\sigma})\right\}, \min \left\{\operatorname{pr}\left(\tilde{K}_{1}\right)(\tilde{s}), \tilde{\Sigma}_{u c}(\tilde{\sigma}), \tilde{M}(\tilde{s} \tilde{\sigma})\right\}\right\} \\
\leq & \min \left\{\operatorname{pr}\left(\tilde{K}_{1}\right)(\tilde{s} \tilde{\sigma}), \operatorname{pr}\left(\tilde{K}_{2}\right)(\tilde{s} \tilde{\sigma})\right\} \\
= & \operatorname{pr}\left(\tilde{K}_{1} \tilde{\cap}_{\tilde{K}_{2}}\right)(\tilde{s} \tilde{\sigma}) .
\end{aligned}
$$


(iii) For any $\tilde{s} \in \tilde{\Sigma}^{*}$ and any $\tilde{\sigma} \in \tilde{\Sigma}$, we have that

$$
\begin{aligned}
\operatorname{pr}\left(\tilde{K}_{1} \tilde{\cap}_{2}\right)(\tilde{s}) & =\sup _{\tilde{s} \in \operatorname{pr}(\tilde{t})}\left(\tilde{K}_{1} \tilde{\cap} \tilde{K}_{2}\right)(\tilde{t}) \\
& =\sup _{\tilde{s} \in \operatorname{pr}(\tilde{t})} \min \left\{\tilde{K}_{1}(\tilde{t}), \tilde{K}_{2}(\tilde{t})\right\} \\
& \leq \min \left\{\sup _{\tilde{s} \in \operatorname{pr}(\tilde{t})} \tilde{K}_{1}(\tilde{t}), \sup _{\tilde{s} \in \operatorname{pr}(\tilde{t})} \tilde{K}_{2}(\tilde{t})\right\} \\
& =\min \left\{\operatorname{pr}\left(\tilde{K}_{1}\right)(\tilde{s}), \operatorname{pr}\left(\tilde{K}_{2}\right)(\tilde{s})\right\} \\
& =\min \left\{\tilde{K}_{1}(\tilde{s}), \tilde{K}_{2}(\tilde{s})\right\} \\
& =\left(\tilde{K}_{1} \tilde{\cap}_{\tilde{K}}\right)(\tilde{s}) \\
& \leq \operatorname{pr}\left(\tilde{K}_{1} \tilde{\cap} \tilde{K}_{2}\right)(\tilde{s}) .
\end{aligned}
$$

The rest of the proof is similar to (ii).

Given a fuzzy language $\tilde{K}$ over set of fuzzy events $\tilde{\Sigma}$, we hope to find the largest fuzzy sublanguage and the smallest prefix-closed fuzzy controllable superlanguage of $\tilde{K}$, such that these languages are controllable with respect to $\tilde{M}$ and $\tilde{\Sigma}_{u c}$. To this end, we denote

$$
\begin{aligned}
& \mathcal{K}(\tilde{K})^{<}=\left\{\tilde{L} \subseteq \tilde{K}: \tilde{L} \in C\left(\tilde{M}, \tilde{\Sigma}_{u c}\right)\right\} \\
& \mathcal{K}(\tilde{K})^{>}=\left\{\tilde{L} \in \mathcal{F}\left(\tilde{\Sigma}^{*}\right): \tilde{K} \subseteq \tilde{L} \subseteq \tilde{M} \text { and } \operatorname{pr}(\tilde{L})=\tilde{L} \text { and } \tilde{L} \in C\left(\tilde{M}, \tilde{\Sigma}_{u c}\right)\right\} \\
& \tilde{K}^{<}=\tilde{\bigcup}_{\tilde{K} \in \mathcal{K}(\tilde{K})^{<}} \tilde{L} \text {, and } \tilde{K}^{>}=\tilde{\bigcap}_{\tilde{K} \in \mathcal{K}(\tilde{K})>} \tilde{L} .
\end{aligned}
$$

Lemma 1. Let $\tilde{M} \in \mathcal{F}\left(\tilde{\Sigma}^{*}\right)$ and $\tilde{\Sigma}_{u c} \in \mathcal{F}(\tilde{\Sigma})$ be the same as those in Definition 1. Suppose $\tilde{K} \in \mathcal{F}\left(\tilde{\Sigma}^{*}\right)$. Then:

(i) $\tilde{K}^{<} \in \mathcal{K}(\tilde{K})^{<}$.

(ii) $\tilde{K}^{>} \in \mathcal{K}(\tilde{K})^{>}$.

Proof: (i) With the proof of Proposition 1 (i) it suffices to show that $\operatorname{pr}\left(\tilde{\cup}_{i \in I} \tilde{L}_{i}\right)=$ $\tilde{\cup}_{i \in I} \operatorname{pr}\left(\tilde{L}_{i}\right)$, where $I$ denotes an index set (perhaps infinite). For any $\tilde{s} \in \tilde{\Sigma}^{*}$, we have

$$
\begin{aligned}
\operatorname{pr}\left(\tilde{\cup}_{i \in I} \tilde{L}_{i}\right)(\tilde{s}) & =\sup _{\tilde{s} \in \operatorname{pr}(\tilde{t})}\left(\tilde{\cup}_{i \in I} \tilde{L}_{i}\right)(\tilde{t}) \\
& =\sup _{\tilde{s} \in \operatorname{pr}(\tilde{t})} \sup _{i \in I} \tilde{L}_{i}(\tilde{t}) \\
& =\sup _{i \in I} \sup _{\tilde{s} \in \operatorname{pr}(\tilde{t})} \tilde{L}_{i}(\tilde{t}) \\
& =\sup _{i \in I} \operatorname{pr}\left(\tilde{L}_{i}\right)(\tilde{s}) \\
& =\left(\tilde{\cup}_{i \in I} \operatorname{pr}\left(\tilde{L}_{i}\right)\right)(\tilde{s}) .
\end{aligned}
$$


(ii) Since $\operatorname{pr}\left(\tilde{L}_{i}\right)=\tilde{L}_{i}$ for each $\tilde{L}_{i} \in \tilde{\mathcal{K}}(\tilde{K})^{>}$, we have that for any $\tilde{s} \in \tilde{\Sigma}^{*}$,

$$
\begin{aligned}
\operatorname{pr}\left(\tilde{K}^{>}\right)(\tilde{s}) & =\operatorname{pr}\left(\tilde{\cap}_{\tilde{L}_{i} \in \mathcal{K}(\tilde{K})>} \tilde{L}_{i}\right)(\tilde{s}) \\
& =\sup _{\tilde{s} \in \operatorname{pr}(\tilde{t})}\left(\tilde{\Pi}_{\tilde{L}_{i} \in \mathcal{K}(\tilde{K})>} \tilde{L}_{i}\right)(\tilde{t}) \\
& =\sup _{\tilde{s} \in \operatorname{pr}(\tilde{t})} \inf _{i} \in \mathcal{K}(\tilde{K})> \\
& \leq \tilde{L}_{i}(\tilde{t}) \\
& =\inf _{\tilde{L}_{i} \in \mathcal{K}(\tilde{K})>} \sup _{\tilde{s} \in \operatorname{pr}(\tilde{t})} \tilde{L}_{i}(\tilde{t}) \\
& =\inf _{\tilde{L}_{i} \in \mathcal{K}(\tilde{K})>} \operatorname{pr}\left(\tilde{L}_{i}\right)(\tilde{s}) \\
& =\inf _{i} \in \mathcal{K}(\tilde{K})^{>} \\
& \tilde{\Pi}_{i}(\tilde{s}) \\
& =\tilde{L}_{i} \in \mathcal{K}(\tilde{K})>(\tilde{s}) \\
& \leq \operatorname{pr}\left(\tilde{L}_{i}\right)(\tilde{s})(\tilde{s})
\end{aligned}
$$

and therefore $\operatorname{pr}\left(\tilde{K}^{>}\right)=\tilde{K}^{>}$. The proof of $\tilde{K}^{>} \in C\left(\tilde{M}, \tilde{\Sigma}_{u c}\right)$ is similar to Proposition 1 (ii).

Clearly, when $\tilde{K} \in C\left(\tilde{M}, \tilde{\Sigma}_{u c}\right)$, we have $\tilde{K}^{<}=\tilde{K}$. Concerning $\tilde{K}^{<}$and $\tilde{K}^{>}$, we further have the following properties.

Proposition 2. Let $\tilde{M} \in \mathcal{F}\left(\tilde{\Sigma}^{*}\right)$ and $\tilde{\Sigma}_{u c} \in \mathcal{F}(\tilde{\Sigma})$ be the same as those in Definition 1. Suppose that $\tilde{K}_{1}, \tilde{K}_{2}$ are fuzzy languages over set $\tilde{\Sigma}$ of fuzzy events. Then:

(i) If $\tilde{K}$ satisfies $\operatorname{pr}(\tilde{K})=\tilde{K}$, then so is $\tilde{K}^{<}$, i.e., $\operatorname{pr}\left(\tilde{K}^{<}\right)=\tilde{K}^{<}$.

(ii) If $\tilde{K}_{1} \subseteq \tilde{K}_{2}$, then $\tilde{K}_{1}^{<} \subseteq \tilde{K}_{2}^{<}$.

(iii) $\left(\tilde{K}_{1} \tilde{\cap} \tilde{K}_{2}\right)^{<} \subseteq \tilde{K}_{1}^{<} \tilde{\cap} \tilde{K}_{2}^{<}$.

(iv) $\left(\tilde{K}_{1} \tilde{\cap}_{\tilde{K}_{2}}\right)^{<}=\left(\tilde{K}_{1}^{<} \tilde{\cap} \tilde{K}_{2}^{<}\right)^{<}$.

(v) If $\operatorname{pr}\left(\tilde{K}_{1}^{<} \tilde{\cap} \tilde{K}_{2}^{<}\right)=\operatorname{pr}\left(\tilde{K}_{1}^{<}\right) \tilde{\cap} \operatorname{pr}\left(\tilde{K}_{2}^{<}\right)$, then $\left(\tilde{K}_{1} \tilde{\cap} \tilde{K}_{2}\right)^{<}=\tilde{K}_{1}^{<} \tilde{\cap} \tilde{K}_{2}^{<}$.

(vi) $\tilde{K}_{1}^{<} \tilde{\cup} \tilde{K}_{2}^{<} \subseteq\left(\tilde{K}_{1} \tilde{\cup} \tilde{K}_{2}\right)^{<}$.

Proof: (i) With Lemma 1 (i) we know that $\tilde{K}^{<} \in \tilde{\mathcal{K}}(\tilde{K})^{<}$, and thus

$$
\operatorname{pr}\left(\tilde{K}^{<}\right) \subseteq \operatorname{pr}(\tilde{K})=\tilde{K} \subseteq \operatorname{pr}(\tilde{K})
$$

which results in $\operatorname{pr}\left(\tilde{K}^{<}\right)=\tilde{K}^{<}$.

(ii) Straightforward. 
(iii) It follows clearly from the definition of $\tilde{K}^{<}$.

(iv) Since $\tilde{K}_{1}^{<} \tilde{\cap} \tilde{K}_{2}^{<} \subseteq \tilde{K}_{1} \tilde{\cap} \tilde{K}_{2}$ from Lemma 1 (i), with (ii) we obtain that $\left(\tilde{K}_{1}^{<} \tilde{\cap} \tilde{K}_{2}^{<}\right)^{<} \subseteq$ $\left(\tilde{K}_{1} \tilde{\cap} \tilde{K}_{2}\right)^{<}$. On the other hand, for any $\tilde{L} \in \mathcal{K}\left(\tilde{K}_{1} \tilde{\cap}_{\tilde{K}_{2}}\right)^{<}$, then $\tilde{L} \in C\left(\tilde{M}, \tilde{\Sigma}_{u c}\right)$ and $\tilde{L} \subseteq$ $\tilde{K}_{1} \tilde{\cap} \tilde{K}_{2}$. Thus $\tilde{L} \subseteq \tilde{K}_{1}$ and $\tilde{L} \subseteq \tilde{K}_{2}$. Since $\tilde{K}_{1}^{<} \subseteq \tilde{K}_{1}$ and $\tilde{K}_{2}^{<} \subseteq \tilde{K}_{2}$ by means of Lemma 1 (i), with the definition of $\tilde{K}^{<}$we obtain that $\tilde{L} \subseteq \tilde{K}_{1}^{<}$and $\tilde{L} \subseteq \tilde{K}_{2}^{<}$, and thus $\tilde{L} \subseteq \tilde{K}_{1}^{<} \tilde{\cap} \tilde{K}_{2}^{<}$. Therefore, for any $\tilde{s} \in \tilde{\Sigma}^{*}$,

$$
\begin{aligned}
\left(\tilde{K}_{1} \tilde{\cap}_{2}\right)^{<}(\tilde{s}) & =\sup \left\{\tilde{L}(\tilde{s}): \tilde{L} \in \mathcal{K}\left(\tilde{K}_{1} \tilde{\cap}_{2}\right)^{<}\right\} \\
& \leq \sup \left\{\tilde{L}(\tilde{s}): \tilde{L} \subseteq \tilde{K}_{1}^{<} \tilde{\cap} \tilde{K}_{2}^{<}, \tilde{L} \in C\left(\tilde{M}, \tilde{\Sigma}_{u c}\right)\right\} \\
& =\left(\tilde{K}_{1}^{<} \tilde{\cap} \tilde{K}_{2}^{<}\right)^{<}(\tilde{s}) .
\end{aligned}
$$

As a result, $\left(\tilde{K}_{1} \tilde{\cap} \tilde{K}_{2}\right)^{<} \subseteq\left(\tilde{K}_{1}^{<} \tilde{\cap} \tilde{K}_{2}^{<}\right)^{<}$, and (iv) is thus proved.

(v) From Proposition 1 (ii) and Lemma 1 (i) it follows that $\tilde{K}_{1}^{<} \tilde{\cap} \tilde{K}_{2}^{<} \in C\left(\tilde{M}, \tilde{\Sigma}_{u c}\right)$. Thus, with (iv) just verified we have that

$$
\tilde{K}_{1}^{<} \tilde{\cap} \tilde{K}_{2}^{<}=\left(\tilde{K}_{1}^{<} \tilde{\cap} \tilde{K}_{2}^{<}\right)^{<}=\left(\tilde{K}_{1} \tilde{\cap} \tilde{K}_{2}\right)^{<}
$$

(vi) For any $\tilde{s} \in \tilde{\Sigma}^{*}$, in light of the definition of $\mathcal{K}(\tilde{K})^{<}$, we have that

$$
\begin{aligned}
\left(\tilde{K}_{1}^{<} \tilde{\cup} \tilde{K}_{2}^{<}\right)(\tilde{s}) & =\max \left\{\tilde{K}_{1}^{<}(\tilde{s}), \tilde{K}_{2}^{<}(\tilde{s})\right\} \\
& =\max \left\{\sup _{\tilde{L}_{1} \in \mathcal{K}\left(\tilde{K}_{1}\right)^{<}} \tilde{L}_{1}(\tilde{s}), \sup _{\tilde{L}_{2} \in \mathcal{K}\left(\tilde{K}_{2}\right)^{<}} \tilde{L}_{2}(\tilde{s})\right\} \\
& \leq \sup _{\tilde{L} \in \mathcal{K}\left(\tilde{K}_{1} \cup \tilde{K}_{2}\right)^{<}} \tilde{L}(\tilde{s}) . \quad \square
\end{aligned}
$$

Proposition 3. Let $\tilde{M} \in \mathcal{F}\left(\tilde{\Sigma}^{*}\right)$ and $\tilde{\Sigma}_{u c} \in \mathcal{F}(\tilde{\Sigma})$ be the same as those in Definition 1 . Suppose that $\tilde{K}_{1}, \tilde{K}_{2}$ are fuzzy languages over set $\tilde{\Sigma}$ of fuzzy events. Then:

(i) If $\tilde{K} \in C\left(\tilde{M}, \tilde{\Sigma}_{u c}\right)$, then $\tilde{K}^{>}=\operatorname{pr}(\tilde{K})$.

(ii) If $\tilde{K}_{1} \subseteq \tilde{K}_{2}$, then $\tilde{K}_{1}^{>} \subseteq \tilde{K}_{2}^{>}$.

(iii) $\left(\tilde{K}_{1} \tilde{\cap} \tilde{K}_{2}\right)^{>} \subseteq\left(\tilde{K}_{1}^{>} \tilde{\cap} \tilde{K}_{2}^{>}\right)^{>}$.

(iv) $\left(\tilde{K}_{1} \tilde{\cup} \tilde{K}_{2}\right)^{>}=\tilde{K}_{1}^{>} \tilde{\cup} \tilde{K}_{2}^{>}$.

Proof: (i) If $\tilde{K} \in C\left(\tilde{M}, \tilde{\Sigma}_{u c}\right)$, then also $\operatorname{pr}(\tilde{K}) \in C\left(\tilde{M}, \tilde{\Sigma}_{u c}\right)$. Together with $\tilde{K} \subseteq \operatorname{pr}(\tilde{K})$ and $\operatorname{pr}(\operatorname{pr}(\tilde{K}))=\operatorname{pr}(\tilde{K})$ we obtain that $\operatorname{pr}(\tilde{K}) \in \mathcal{K}(\tilde{K})^{>}$since $\tilde{M}=\operatorname{pr}(\tilde{M})$. Thus, $\tilde{K}^{>} \subseteq$ $\operatorname{pr}(\tilde{K})$. Contrarily, for any $\tilde{L} \in \mathcal{K}(\tilde{K})^{>}$, then $\operatorname{pr}(\tilde{L})=\tilde{L}$ and we thus have

$$
\operatorname{pr}(\tilde{K}) \subseteq \operatorname{pr}(\tilde{L})=\tilde{L} .
$$


Therefore $\operatorname{pr}(\tilde{K}) \subseteq \tilde{\bigcap}_{\tilde{L} \in \mathcal{K}(\tilde{K})>} \tilde{L}=\tilde{K}^{>}$.

The demonstrations of (ii) and (iii) are clear, so, we here omit the details.

(iv) Due to $\tilde{K}_{1} \subseteq \tilde{K}_{1} \tilde{\cup} \tilde{K}_{2}$ and $\tilde{K}_{2} \subseteq \tilde{K}_{1} \tilde{\cup} \tilde{K}_{2}$, we have

$$
\mathcal{K}\left(\tilde{K}_{1} \tilde{\cup} \tilde{K}_{2}\right)^{>} \subseteq \mathcal{K}\left(\tilde{K}_{1}\right)^{>} \tilde{\cup} \mathcal{K}\left(\tilde{K}_{2}\right)^{>}
$$

Thus,

$$
\tilde{K}_{1}^{>} \tilde{\cup} \tilde{K}_{2}^{>} \subseteq\left(\tilde{K}_{1} \tilde{\cup} \tilde{K}_{2}\right)^{>} .
$$

On the other hand, for any $\tilde{s} \in \tilde{\Sigma}^{*}$, suppose that

$$
\left(\tilde{K}_{1} \tilde{\cup} \tilde{K}_{2}\right)^{>}(\tilde{s})=\inf _{\tilde{L} \in \mathcal{K}\left(\tilde{K}_{1} \cup \tilde{K} \tilde{K}_{2}\right)^{>}} \tilde{L}(\tilde{s})>t
$$

for $t \in[0,1)$. Then we claim that

$$
\left(\tilde{K}_{1}^{>} \tilde{\cup} \tilde{K}_{2}^{>}\right)(\tilde{s})>t
$$

otherwise, $\tilde{K}_{1}^{>}(\tilde{s}) \leq t$ and $\tilde{K}_{2}^{>}(\tilde{s}) \leq t$, from which together with the definition of $\left(\tilde{K}_{1}^{>} \tilde{\cup} \tilde{K}_{2}^{>}\right)(\tilde{s})$, i.e.,

$$
\left(\tilde{K}_{1}^{>} \tilde{\cup} \tilde{K}_{2}^{>}\right)(\tilde{s})=\max \left\{\inf _{\tilde{L}_{1} \in \mathcal{K}\left(\tilde{K}_{1}\right)>} \tilde{L}_{1}(\tilde{s}), \inf _{\tilde{L}_{2} \in \mathcal{K}\left(\tilde{K}_{2}\right)^{>}} \tilde{L}_{2}(\tilde{s})\right\},
$$

it follows that for any $\varepsilon>0$, there exist $\tilde{L}_{1} \in \mathcal{K}\left(\tilde{K}_{1}\right)^{>}$and $\tilde{L}_{2} \in \mathcal{K}\left(\tilde{K}_{2}\right)^{>}$such that $\tilde{L}_{1}(\tilde{s})<t+\varepsilon$ and $\tilde{L}_{2}(\tilde{s})<t+\varepsilon$. Due to $\operatorname{pr}\left(\tilde{L}_{1} \tilde{\cup} \tilde{L}_{2}\right)=\operatorname{pr}\left(\tilde{L}_{1}\right) \tilde{\cup} \operatorname{pr}\left(\tilde{L}_{2}\right)$ and Proposition 1 (i), it follows that $\tilde{L}_{1} \tilde{\cup} \tilde{L}_{2} \in \mathcal{K}\left(\tilde{L}_{1} \tilde{\cup} \tilde{L}_{2}\right)^{>}$. Consequently,

$$
\begin{aligned}
\left(\tilde{L}_{1} \tilde{\cup} \tilde{L}_{2}\right)^{>}(\tilde{s}) & \leq\left(\tilde{L}_{1} \tilde{\cup} \tilde{L}_{2}\right)(\tilde{s}) \\
& =\max \left\{\tilde{L}_{1}(\tilde{s}), \tilde{L}_{2}(\tilde{s})\right\} \\
& <t+\varepsilon .
\end{aligned}
$$

Since $\varepsilon$ is arbitrary, we have $\left(\tilde{L}_{1} \tilde{\cup} \tilde{L}_{2}\right)^{>}(\tilde{s}) \leq t$, which contradicts the assumption of Eq. (20). Therefore, Eq. (21), i.e., $\left(\tilde{L}_{1} \tilde{\cup} \tilde{L}_{2}\right)^{>}(\tilde{s})>t$ holds true. As a consequence, $\left(\tilde{K}_{1} \tilde{\cup}_{K_{2}}\right)^{>}(\tilde{s}) \leq$ $\left(\tilde{K}_{1}^{>} \tilde{\cup} \tilde{K}_{2}^{>}\right)(\tilde{s})$, and therefore $\left(\tilde{K}_{1} \tilde{\cup} \tilde{K}_{2}\right)^{>} \subseteq \tilde{K}_{1}^{>} \tilde{\cup} \tilde{K}_{2}^{>}$is proved.

\section{Concluding Remarks}

Fuzzy DESs initiated by Lin and Ying $[19,18]$ may conform more to human's perception when coping with some real-world problems, especially in biomedical applications and likely 
in traffic systems (the changes of traffic light are controlled in terms of the length of queues of vehicles, in which length is a fuzzy concept). With a desire to provide certain foundation for the applications of fuzzy DESs, in this paper we further developed fuzzy DESs by dealing with supervisory control issue of fuzzy DESs. The technical contributions are mainly as follows: (i) we reformulated the parallel composition of crisp DESs, and defined the parallel composition of fuzzy DESs that is equivalent to that in [19]; max-min and max-product automata for modeling DESs are considered; (ii) we dealt with a number of basic problems in supervisory control of fuzzy DESs, and demonstrated controllability theorem and nonblocking controllability theorem of fuzzy DESs, and thus obtained the conditions for the existence of supervisors in fuzzy DESs; (iii) after analyzing the complexity for presenting a uniform criterion for testing the fuzzy controllability condition of fuzzy DESs modeled by max-product automata, we particularly presented in detail a computing flow for checking the fuzzy controllability condition of fuzzy DESs modeled by max-min automata, and by means of this method we can search for all possible fuzzy states reachable from initial fuzzy state in max-min automata; as well, the fuzzy $n$-controllability condition was introduced for some realistic control problems; (iv) a number of examples serving to illustrate the applications of the obtained methods and theorems were described; (v) some basic properties related to supervisory control of fuzzy DESs were discussed, which include the existence of supremal fuzzy controllable sublanguage and infimal prefix-closed fuzzy controllable superlanguage of a given fuzzy noncontrollable sublanguage.

As was known [1], in classical DESs, under certain condition we can check within finite number of steps whether the controllability condition holds by utilizing the finiteness of states in crisp finite automata, but the infiniteness of fuzzy states in fuzzy DESs modeled by maxproduct automata gives rise to considerable complexity for formulating a uniform fashion to check the fuzzy controllability conditions. However, when fuzzy DESs are modeled by maxmin automata, we have derived a uniform criterion (computing flow) to check this condition. This is a very important result in supervisory control of fuzzy DESs. Notably, our methods also apply to testing supervisory control condition of crisp DESs. Therefore, it has been seen that there are some essential differences between fuzzy DESs and crisp DESs, and the computing processes in fuzzy DESs are also more complicated. A significant issue is to give some efficient algorithms in fuzzy DESs such as constructing efficient algorithms to check the fuzzy controllability conditions presented in Theorems 1 and 2, on the basis of the computing flow described in this paper. 
With the results obtained in Ref. [19] and this paper, a further issue also worthy of consideration is to deal with supervisory control of fuzzy DESs modeled via max-min automata under partial observation, which includes how to establish controllability and observability theorem of fuzzy DESs, and how to deal with decentralized supervisory control of fuzzy DESs. Another important modeling approach to fuzzy DESs, that is, fuzzy DESs modeled by fuzzy Petri nets [15], has not been investigated, and we deem it a significant research direction. Finally, it is worth indicating that computing with words, as a methodology, advocated by Zadeh and others $[38,39,40,37,36]$, may play a useful role in the further exploration of fuzzy DESs. These problems will be considered in subsequent work.

\section{Acknowledgments}

The author wishes to thank L. O. Hall, and the Associate Editor, as well as the anonymous reviewers for their invaluable suggestions and comments that greatly helped to improve the quality of this paper.

\section{References}

[1] C. G. Cassandras and S. Lafortune, Introduction to Discrete Event Systems. Boston, MA: Kluwer, 1999.

[2] R. David and H. Alla, Petri Nets and Grafcet: Tools for modelling discrete event systems. Englewood Cliffs, NJ: Prentice-Hall, 1992.

[3] D. Dubois and H. Prade, Fuzzy Sets and Systems: Theory and Applications, New York: Academic, 1980.

[4] C. Giles, C. Omlin, and K. K. Thornber, "Equivalence in knowledge representation: automata, recurrent neural networks, and dynamical fuzzy systems," in Proc. of the IEEE, vol. 87, pp. 1623-1640, 1999.

[5] S. Hikmet, "Fuzzy command grammars for intelligent interface design," IEEE Trans. Syst., Man, Cybern., vol. 22, no. 5, pp. 1124-1131, 1992. 
[6] J. W. Huang, C. M. Held, and R. J. Roy, "Hemodynamic Management with Multiple Drug using Fuzzy Logic," in Fuzzy and Neuro-Fuzzy Systems in Medicine, H. N. Teodoresch, A. Kandel, and L. C. Jain, Eds. Boca Raton: CRC Press, 1999, pp. 319-340.

[7] L. E. Holloway, B. H. Krogh, and A. Giua, "A Suvery of Petri Net methods for Controlled Discrete Event Systems," Discrete Event Systems: Theory and Applications, vol. 7, no. 2, pp. 151-190, 1997.

[8] M. Heymann and F. Lin, "Discrete-Event Control of Nondeterministic Systems," IEEE Trans. Automat. Contr., vol. 43, no. 1, pp. 3-17, Jan. 1998.

[9] N. Honda, M. Nasu, and S. Hirose, "F-recognition of fuzzy languages," in Fuzzy Automata and Decision Processes, M. M. Gupta, G. N. Saridis, and B. R. Gaines, Eds. Amsterdam: North-Holland, 1977, pp. 149-168.

[10] J. E. Hopcroft and J. D. Ullman, Introduction to Automata Theory, Languages, and Computation, Reading, MA: Addision-Wesley, 1979.

[11] R. Kumar and V. K. Garg, Modeling and Control of Logical Discrete Event Systems, Boston, MA: Kluwer, 1995.

[12] A. Kandel and S. C. Lee, Fuzzy Switching and Automata: Theory and Applications. New York: Russak, 1979.

[13] G. J. Klir and B. Yuan, Fuzzy Sets and Fuzzy Logic: Theory and Applications. Englewood Cliffs, NJ: Prentice-Hall, 1995.

[14] L. L. Leape, "The Preventability of Medical Injury", in Human Error in Medicine, M. S. Bogner, Ed. Hillsdale, NJ: Lawrence Erlbaum Associates, 1994, pp. 13-25.

[15] C. G. Looney, "Fuzzy Petri Nets for Rule-Based Decisionmaking," IEEE Trans. Syst., Man, Cybern., vol. 18, no. 1, pp. 178-183, 1988.

[16] Y. Li, F. Lin, and Z. Lin, "Supervisory Control of Probabilistic Discrete-Event Systems with Recovery," IEEE Trans. Automat. Contr., vol. 44, no. 10, pp. 1971-1975, Oct. 1999.

[17] F. Lin and W. M. Wonham, "On observability of discrete event systems," Inform. Sci., vol. 44, no. 3, pp. 173-198, 1988. 
[18] F. Lin and H. Ying, "Fuzzy discrete event systems and their observability," in Pro. Joint Int. Conf. 9th Int. Fuzzy Systems Assoc. World Congr. 20th North Amer. Fuzzy Inform. Process. Soci., Vancouver, BC, Canada, July 25-28, 2001.

[19] F. Lin and H. Ying, "Modeling and Control of Fuzzy Discrete Event Systems," IEEE Trans. Syst., Man, Cybern. B, vol. 32, no. 4, Aug. pp. 408-415, 2002.

[20] E. T. Lee and L. A. Zadeh, "Note on fuzzy languages," Inform. Sci., vol. 1, pp. 421-434, 1969.

[21] J. N. Mordeson and D. S. Malik, Fuzzy Automata and Languages: Theory and applications, Boca Raton, London: Chapman \& Hall/CRC, 2002.

[22] J. L. Peterson, Petri Net Theory and the Modeling of Systems. Englewood Cliffs, NJ: Prentice-Hall, 1981.

[23] D. W. Qiu, "Automata theory based on completed residuated lattice-valued logic (I)," Science in China (F), vol. 44, no. 6, pp. 419-429, 2001.

[24] D. W. Qiu, "Automata theory based on completed residuated lattice-valued logic (II)," Science in China (F), vol. 45, no. 6, pp. 442-452, 2002.

[25] D. W. Qiu, "Characterizations of Fuzzy Finite Automata," Fuzzy Sets Syst., vol. 141, no. 3, pp. 391-414, 2004.

[26] R. J. Ramadge and W. M. Wonham, "Supervisory control of a class of discrete event processes," SIAM J. Contr. Optim., vol. 25, no. 1, pp. 206-230, Jan. 1987.

[27] R. J. Ramadge and W. M. Wonham, "The control of discrete event systems," in Proc. of the IEEE, vol. 77, no. 1, 1989, pp. 81-98.

[28] E. S. Santos, "Maxmin automata," Inform. Contr., vol. 13, pp. 363-377, 1968.

[29] E. S. Santos, "Regular fuzzy expressions," in Fuzzy Automata and Decision Processes, M. M. Gupta, G. N. Saridis, and B. R. Gaines, Eds. Amsterdam: North-Holland, 1977, pp. 169-175.

[30] F. Steimann and K. P. Adlassning, "Clinical monitoring with fuzzy automata," Fuzzy Sets Syst., vol. 61, pp. 37-42, 1994. 
[31] J. G. Thistle, "Supervisory control of discrete event systems," Mathematical and Computer Modelling, vol. 23, no. 11/12, pp.25-53, 1996.

[32] M. G. Thomason and P. N. Marinos, "Deterministic acceptors of regular fuzzy languages," IEEE Trans. Syst., Man, Cybern., vol. 4, pp. 228-230, 1974.

[33] F. A. Unal and E. Khan, "A fuzzy finite state machine implementation based on a neural fuzzy system," in Proc. 3rd Int. Conf. Fuzzy Syst., vol. 3, 1994, pp. 1749-1754.

[34] W. G. Wee, "On generalizations of adaptive algorithm and application of the fuzzy sets concept to pattern classification," Ph.D. dissertation, Purdue University, June, 1967.

[35] W. M. Wonham and R. J. Ramadge, "On the supremal controllable sublanguage of a given language," SIAM J. Contr. Optim., vol. 25, no. 3, pp. 637-659, May 1987.

[36] H. Wang and D. W. Qiu, "Computing With Words via Turing Machines: A Formal Approach," IEEE Trans. Fuzzy Syst., vol. 11, no. 6, pp. 742-753, 2003.

[37] M. S. Ying, "A Formal Model of Computing with words," IEEE Trans. Fuzzy Syst., vol. 10, no. 5, pp. 640-652, 2002.

[38] L. A. Zadeh, "Fuzzy Logic=Computing with Words," IEEE Trans. Fuzzy Syst., vol. 4, no. 2, pp. 103-111, 1996.

[39] L. A. Zadeh, "Toward a Logic of Perceptions Based on Fuzzy Logic," in Discovering the World with Fuzzy Logic, V. Novak, I. Perfilieva, Eds. New York: Physica-Verlag, 2000, pp. 4-28.

[40] L. A. Zadeh and J. Kacprzyk, Computing With Words in Information/Intelligent Systems, ser. Studies in Fuzziness and Soft Computing. Berlin, Germany: Physica-Verlag, 1999, vol. 33/34. 